\title{
PARTITIONS FOR SPECTRAL (FINITE) VOLUME RECONSTRUCTION IN THE TETRAHEDRON
}

\author{
By
}

\author{
Qian-Yong Chen
}

IMA Preprint Series \# 2035

(April 2005)

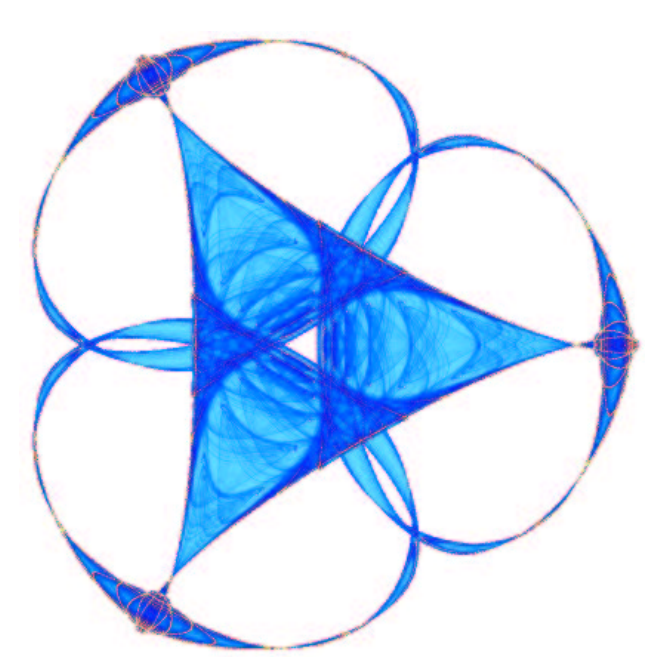

INSTITUTE FOR MATHEMATICS AND ITS APPLICATIONS UNIVERSITY OF MINNESOTA

514 Vincent Hall 206 Church Street S.E. Minneapolis, Minnesota 55455-0436 Phone: 612/624-6066 Fax: 612/626-7370 URL: http://www.ima.umn.edu 


\title{
Partitions for Spectral (Finite) Volume Reconstruction in the Tetrahedron
}

\author{
Qian-Yong Chen*
}

\begin{abstract}
In this paper, we compute partitions of the tetrahedron for up to the fourth-order spectral volume reconstruction. Certain optimization is made to these partitions and previously obtained partitions of lower dimensional simplex. These optimized partitions have the smallest Lebesgue constants among currently known spectral volume partitions.
\end{abstract}

${ }^{*}$ Institute for Mathematics and its Applications, University of Minnesota, Minneapolis, MN 55455 (qchen@ima.umn.edu). 


\section{Introduction}

Spectral volume reconstruction is a key element of the recently proposed spectral volume method $[10,11]$ for hyperbolic conservation laws. Analogous to the well known fact that the quality of polynomial interpolation depends on the interpolation points set, the quality of spectral volume reconstruction in the simplex is determined by the partition of the simplex [3]. Since the development of the spectral volume method, some research has been done on the partition generation. For example, by following the idea of Chen and Babuška [1, 2], Wang and Liu computed the so called mean $L^{2}$ optimal partitions for up to the seventh order spectral volume reconstruction of the one-dimensional simplex [9]. Several systematic techniques based on the Voronoi diagram and its variants have also been developed in [3] for both the one and two-dimensional simplex. More recently, a linear partition and a quadratic partition of the tetrahedron were constructed in [7].

However, partitions for high order spectral volume reconstruction on the tetrahedron are still unavailable. In this paper we compute up to the fourth-order partitions of the three-dimensional simplex, $S^{3}$. These partitions are based on the idea we proposed in [3], i.e., building the partition through extensive use of the geometry structure of the interpolation points in the simplex, such as the symmetry and layering structure. The idea can be extended to generate higher order partitions of the tetrahedron. Optimization within the framework of building these partitions is also made for these partitions and the previously obtained partitions of the one and two-dimensional simplex.

Denote $\mathbf{P}^{n}\left(S^{3}\right)$ as the space of polynomials of degree up to $n$ in three variables. The dimension of this approximation space is

$$
N_{n}=\operatorname{dim} \mathbf{P}^{n}\left(S^{3}\right)=\left(\begin{array}{c}
3+n \\
3
\end{array}\right)=\frac{(n+1)(n+2)(n+3)}{6} .
$$

$N=N_{n}$ and $\mathbf{P}^{n}=\mathbf{P}^{n}\left(S^{3}\right)$ will be used to simplify the notations if there is no confusion. Assume $\left\{p_{1}(x, y, z), \cdots, p_{N}(x, y, z)\right\}$ forms a complete basis of $\mathbf{P}^{n}\left(S^{3}\right)$.

Given any continuous function $u(x, y, z)$ on $S^{3}$, i.e., $u \in C\left(S^{3}\right)$, the computation of its $n$-th order spectral volume reconstruction on $S^{3}$ consists of two steps:

1. Construct a partition $\Pi_{n}$ of $S^{3}$ with $N$ non-overlapping sub-cells (only polyhedron sub-cells 
are considered):

$$
S^{3}=C_{1} \cup \cdots \cup C_{N}
$$

2. Find a projection $\mathcal{I}_{\Pi_{n}} u=\sum_{i=1}^{N} a_{i} p_{i}(x, y, z) \in \mathbf{P}^{n}$, which shares the same average as $u$ on all the sub-cells, i.e.,

$$
\frac{1}{V_{i}} \int_{C_{i}}\left(\mathcal{I}_{\Pi_{n}} u\right) d V=\frac{1}{V_{i}} \int_{C_{i}} u(x, y, z) d V, \quad i=1, \cdots, N
$$

where $V_{i}$ denotes the area of sub-cell $C_{i}$.

Denote $\bar{u}_{i}$ as the average of $u(x, y, z)$ over sub-cell $C_{i}$, i.e.,

$$
\bar{u}_{i}=\frac{1}{V_{i}} \int_{C_{i}} u(x, y, z) d V, \quad i=1, \cdots, N .
$$

Rewrite the system (1) into a matrix form: $\mathbf{A a}=\mathbf{u}$ with $\mathbf{a}=\left(a_{1}, \cdots, a_{N}\right)^{T}$ and $\mathbf{u}=\left(\bar{u}_{1}, \cdots, \bar{u}_{N}\right)^{T}$. The reconstruction matrix $\mathbf{A}$ takes the form

$$
\mathbf{A}=\left(\begin{array}{ccc}
\frac{1}{V_{1}} \int_{C_{1}} p_{1}(x, y, z) d V & \cdots & \frac{1}{V_{1}} \int_{C_{1}} p_{N}(x, y, z) d V \\
\cdots & \cdots & \cdots \\
\frac{1}{V_{N}} \int_{C_{N}} p_{1}(x, y, z) d V & \cdots & \frac{1}{V_{N}} \int_{C_{N}} p_{N}(x, y, z) d V
\end{array}\right)
$$

Assuming the reconstruction matrix is nonsingular, the projection $\mathcal{I}_{\Pi_{n}} u$ can be expressed in the Lagrange form, $\mathcal{I}_{\Pi_{n}} u=\sum_{i=1}^{N} \bar{u}_{i} L_{i}(x, y, z)$, where the cardinal basis functions $\mathbf{L}=\left(L_{1}, \cdots, L_{N}\right)=$ $\left(p_{1}, \cdots, p_{N}\right) \mathbf{A}^{-1}$.

Then we equip the space $\mathbf{P}^{n}$ and $C\left(S^{3}\right)$ with an $L^{\infty}$ norm (supremum-norm, denoted as $\|\cdot\|$ ) and the induced functional norm

$$
\left\|\mathcal{I}_{\Pi_{n}}\right\|=\sup _{\|u\| \neq 0} \frac{\left\|\mathcal{I}_{\Pi_{n}} u\right\|}{\|u\|} .
$$

Since $\left|\bar{u}_{i}\right| \leq\|u\|$ for $i=1, \cdots, N$, one can show that

$$
\left\|\mathcal{I}_{\Pi_{n}}\right\|=\max _{(x, y, z) \in S^{3}} \sum_{i=1}^{N}\left|L_{i}(x, y, z)\right|
$$

From the linearity of the projection operator $\mathcal{I}_{\Pi_{n}}$ and the fact that $\mathcal{I}_{\Pi_{n}} f=f, \forall f \in \mathbf{P}^{n}\left(S^{3}\right)$, it is easy to verify that the error of spectral volume reconstruction can be bounded as

$$
\left\|u-\mathcal{I}_{\Pi_{n}} u\right\| \leq\left(1+\Lambda\left(\Pi_{n}\right)\right)\left\|u-u^{*}\right\|
$$


where $u^{*}$ is the optimal approximating polynomial whose existence is guaranteed by the continuity of $u(x, y, z)[4]$. And

$$
\Lambda\left(\Pi_{n}\right)=\left\|\mathcal{I}_{\Pi_{n}}\right\|=\max _{(x, y) \in S^{3}} \sum_{i=1}^{N}\left|L_{i}(x, y, z)\right|
$$

is called the Lebesgue constant of the operator $\mathcal{I}_{\Pi_{n}}$.

According to (3), the partitions with small Lebesgue constants are preferred. It is rather difficult to directly build good high order partitions, specially for three dimensional spectral volume reconstructions, because there are too many parameters such as the position of points, the number of edges for each sub-cell and the topology of the sub-cells. For another reconstruction problem, the polynomial interpolation, several almost optimal sets have been obtained (see $[8,5,1,2]$ and reference therein). At a glimpse, it seems that the methodology of $[1,2]$ can be used to optimize the spectral volume partition. However, as shown in [9], the mean $L^{2}$ optimal partitions are even not very satisfactory for the one-dimensional case. So in this paper we do not compute the mean $L^{2}$ optimal partitions. Instead, we only compute partitions based on the polynomial interpolation points, and try to optimize these partitions within the framework of constructing them. In addition, we try to minimize the number of total faces of the partition whenever it is possible.

The rest of the paper includes two sections. In Sec. 2, we describe the algorithm of computing up to the fourth order partition of the tetrahedron. Section 3 is devoted to the optimization of the partitions in Sec. 2 and the partitions of the lower dimensional simplex developed in [3]. 


\section{Partitions of the Tetrahedron}

In this section, we propose an algorithm to compute symmetric partitions for the spectral volume reconstruction in the tetrahedron. Similar to the technique developed in [3] for the lower dimensional simplex, this algorithm exploits the geometry structure of interpolation points on the tetrahedron (points from [6] are used in this paper). The algorithm is described in a recursive fashion in the sense of high order partitions being based on lower order partitions. In specific, when building high order partitions, we first group all the interpolation points except those on a single tetrahedron face into a new points set. Then we construct some sub-cells from the new points set with the algorithm for the one-order lower partition. We will explain it in detail in the following.

The symmetry property of the partitions is extensively used in the algorithm. But unlike polynomial interpolation, we cannot first compute the possible number of different symmetric points such as four-fold or six-fold symmetric points, as the authors did in [2]. It is because the total number of vertices for any order partition is not a fixed number, which is a direct consequence of the fact that a face can have any number $(\geq 3)$ of vertices. For a similar reason, the total number of faces is also not a fixed number for a given order partition of the tetrahedron. So in the following, we will try to minimize, besides the Lebesgue constant, the number of faces as that is proportional to the work load of the spectral volume method $[11,10]$.

\subsection{The First Order Partition}

Our first order partition is the same as that given in [7]. But we describe our algorithm within a more general setting so that the algorithm can be used to generate higher order partitions.

Follow the idea of [3], we build the partition from the polynomial interpolation points on the tetrahedron in a certain way such that each sub-cell contains an input point. For the first order partition, the input points are simply the vertices of the tetrahedron. So a sub-cell is needed for each vertex of the tetrahedron. The four sub-cells can be computed in the exactly same way because of symmetry. Hence we only describe how to construct the sub-cell for one vertex. Figure 1 shows the sub-cell for vertex $A$ in tetrahedron $A B C D$ (point $D$ is behind the scene). This sub-cell consists of 


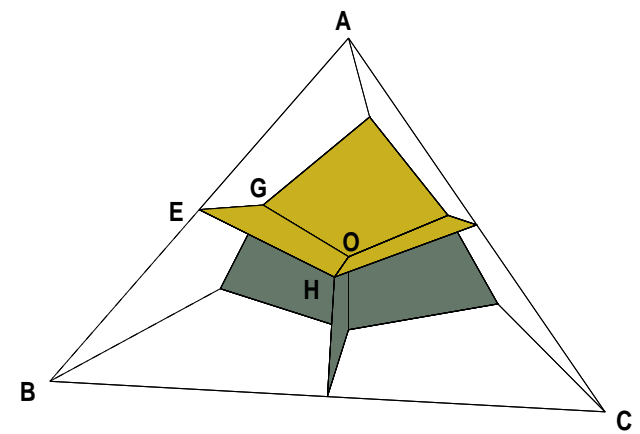

Figure 1: First order partition of the tetrahedron.

three interior 'faces' and three faces which are on the faces of the tetrahedron. Again by symmetry, the three interior faces can be constructed in a similar way. One such interior face is $E H O G$, in which $O$ is inside the tetrahedron, $E$ is on the edge $A B$, and $G$ and $H$ are on the face $A B D$ and $A B C$ respectively. In this face, we require that point $G$ has the same barycenter coordinates in $\triangle A B D$ as the point $H$ in $\triangle A B C$. Moreover, as shown in Fig. 1, the three remaining faces will be fixed after building the interior faces. So it suffices to specify how to choose points $O, E$ and $G$, in order to compute the sub-cell for vertex $A$, thus the whole first order partition. In the following, bold symbols represent the coordinates of points in column vector form.

Note that the points $E, H, O$ and $G$ are not always coplanar. We choose the points $O, E$ and $G$ with form

$$
\left\{\begin{array}{l}
\mathbf{O}=r \mathbf{B}+r \mathbf{C}+r \mathbf{D}+(1-3 r) \mathbf{A} \\
\mathbf{E}=s \mathbf{B}+(1-s) \mathbf{A} \\
\mathbf{G}=t \mathbf{B}+t \mathbf{D}+(1-2 t) \mathbf{A}
\end{array}\right.
$$

where $0<r, s, t<1$. For the first order partition, we have $r=1 / 4, s=1 / 2$ and $t=1 / 3$ according to the symmetry property. So $O, E$ and $G$ are the mass center of the tetrahedron $A B C D$, edge $A B$ and face $A B D$ respectively. The partition consists of four hexahedrons, each of which has six quadrilateral faces as shown in Fig. 1. (From Lemma 1, one can verify that the points $O, G, E$ and $H$ are coplanar.) The data set of the partition is given in Table 1-3. The Lebesgue constant for such partition is $95 / 26$ (see [7]). 
Lemma 1 The points $O, G, E$ and $H$ are coplanar if and only if $(s t+r t-2 r s)=0$.

Proof: According to symmetry, $\mathbf{H}=t \mathbf{B}+t \mathbf{C}+(1-2 t) \mathbf{A}$. So

$$
\left(\begin{array}{llll}
\mathbf{O} & \mathbf{G} & \mathbf{E} & \mathbf{H}
\end{array}\right)=\left(\begin{array}{llll}
\mathbf{A} & \mathbf{B} & \mathbf{C} & \mathbf{D}
\end{array}\right)\left(\begin{array}{cccc}
1-3 r & 1-2 t & 1-s & 1-2 t \\
r & t & s & t \\
r & 0 & 0 & t \\
r & t & 0 & 0
\end{array}\right) .
$$

The points $O, G, E$ and $H$ are coplanar if and only if the above matrix is singular. The determinant of the above matrix is equal to $t(s t+r t-2 r s)$, which finishes the proof.

Table 1: The first order partition of the tetrahedron: the barycenter coordinates of the vertices.

\begin{tabular}{c|cccc}
\hline$i$ & & & & \\
\hline 1 & 0.0000000000 & 0.0000000000 & 1.0000000000 & 0.0000000000 \\
2 & 0.0000000000 & 0.0000000000 & 0.5000000000 & 0.5000000000 \\
3 & 0.0000000000 & 0.5000000000 & 0.5000000000 & 0.0000000000 \\
4 & 0.0000000000 & 0.3333333333 & 0.3333333333 & 0.3333333333 \\
5 & 0.0000000000 & 1.0000000000 & 0.0000000000 & 0.0000000000 \\
6 & 0.0000000000 & 0.5000000000 & 0.0000000000 & 0.5000000000 \\
7 & 0.0000000000 & 0.0000000000 & 0.0000000000 & 1.0000000000 \\
8 & 0.2500000000 & 0.2500000000 & 0.2500000000 & 0.2500000000 \\
9 & 0.3333333333 & 0.3333333333 & 0.3333333333 & 0.0000000000 \\
10 & 0.3333333333 & 0.0000000000 & 0.3333333333 & 0.3333333333 \\
11 & 0.3333333333 & 0.3333333333 & 0.0000000000 & 0.3333333333 \\
12 & 0.5000000000 & 0.5000000000 & 0.0000000000 & 0.0000000000 \\
13 & 0.5000000000 & 0.0000000000 & 0.5000000000 & 0.0000000000 \\
14 & 0.5000000000 & 0.0000000000 & 0.0000000000 & 0.5000000000 \\
15 & 1.0000000000 & 0.0000000000 & 0.0000000000 & 0.0000000000 \\
\hline
\end{tabular}

\subsection{The Second Order Partition}

Second-order polynomial interpolation on the tetrahedron needs ten interpolation points. We choose four tetrahedron vertices and six tetrahedron edge middle points as the interpolation points (see Fig. 2). For the four tetrahedron vertices, we construct a sub-cell for each of them as we did for the first order partition in the last section. For example, when building the sub-cell for vertex $A$, we treat $A$ as a vertex of a small tetrahedron $A I J H$ (Fig. 2), and employ the algorithm described 
Table 2: The first order partition of the tetrahedron: the faces. Each row lists the indices of vertices for one face. $M$ denotes the number of vertices of each individual face.

\begin{tabular}{cc|cccc}
\hline$i$ & $M$ & & & & \\
\hline 1 & 4 & 9 & 13 & 15 & 12 \\
2 & 4 & 10 & 14 & 15 & 13 \\
3 & 4 & 11 & 14 & 15 & 12 \\
4 & 4 & 8 & 11 & 14 & 10 \\
5 & 4 & 8 & 11 & 12 & 9 \\
6 & 4 & 8 & 10 & 13 & 9 \\
7 & 4 & 1 & 13 & 10 & 2 \\
8 & 4 & 1 & 13 & 9 & 3 \\
9 & 4 & 1 & 3 & 4 & 2 \\
10 & 4 & 3 & 9 & 8 & 4 \\
11 & 4 & 2 & 10 & 8 & 4 \\
12 & 4 & 3 & 5 & 6 & 4 \\
13 & 4 & 5 & 12 & 11 & 6 \\
14 & 4 & 3 & 9 & 12 & 5 \\
15 & 4 & 4 & 8 & 11 & 6 \\
16 & 4 & 6 & 11 & 14 & 7 \\
17 & 4 & 2 & 7 & 6 & 4 \\
18 & 4 & 2 & 10 & 14 & 7 \\
\hline
\end{tabular}

Table 3: The first order partition of the tetrahedron: the sub-cells. Each row lists the indices of faces of one sub-cell. $M$ denotes the number of faces of each individual sub-cell.

\begin{tabular}{cc|cccccc}
\hline$i$ & $M$ & & & & & & \\
\hline 1 & 6 & 1 & 2 & 3 & 4 & 5 & 6 \\
2 & 6 & 7 & 8 & 9 & 10 & 11 & 6 \\
3 & 6 & 12 & 13 & 14 & 5 & 10 & 15 \\
4 & 6 & 16 & 17 & 18 & 11 & 4 & 15 \\
\hline
\end{tabular}



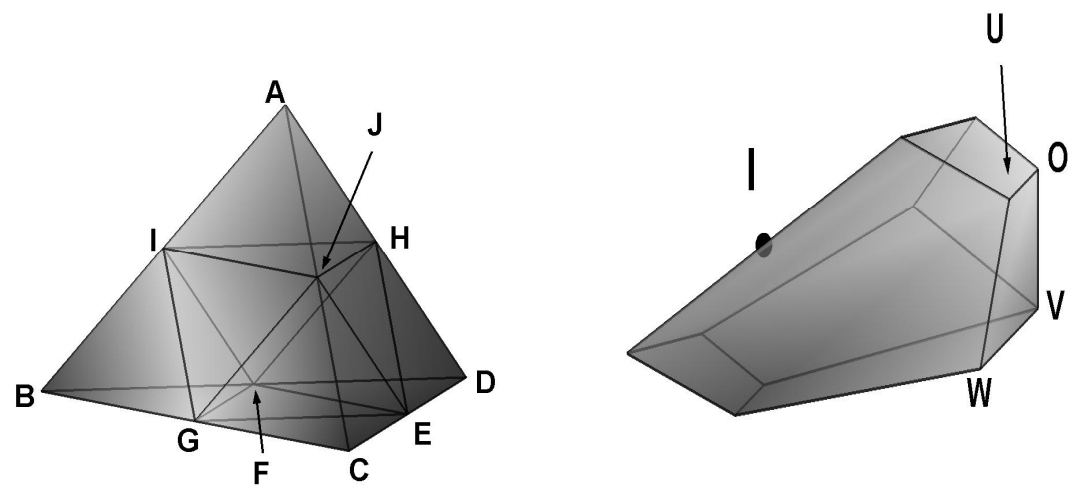

Figure 2: The second order partition. Left: a tetrahedron with input points; Right: the sub-cell that includes point $I$.

in the last section to construct the sub-cell containing $A$. Note that the three parameters $r, s$ and $t$ are not constant this time. We consider only the parameters which ensure that each of these four sub-cells has six quadrilateral faces (i.e., condition of Lemma 1 is satisfied). In Sec. 3.3, we will vary $r, s, t$ with the above constraint to minimize the Lebesgue constant of the partition.

For the six remaining points, it is enough to explain how to compute the sub-cell for one point (e.g., point $I$ ) as we will build the other five sub-cells in a symmetric way. In our second order partition, the sub-cell (Fig. 2) for point $I$ has eight faces: two quadrilateral faces, two pentagon faces, and four more symmetric quadrilateral 'faces'. Among those faces, the two quadrilateral faces also separately belong to the sub-cell including point $A$ and $B$. The two pentagon faces are on the tetrahedron face $A B C$ and $A B D$ respectively, and they will be fixed after building the four symmetric faces. In Fig. 2, we mark one of these four faces as $U O V W$. In this face, the point $U$ is also on the triangle face $A I J$, and $O$ is inside the small tetrahedron $A I J H$. In fact, these two points have already been specified when building the sub-cell for vertex $A$. So the only remaining work is to choose points $W$ and $V$. We put point $W$ inside the triangle $I J G$ and $V$ inside the octahedron $I J H E F G$. In particular, we choose

$$
\left\{\begin{array}{l}
\mathbf{W}=\frac{\mathbf{I}+\mathbf{J}+\mathbf{G}}{3} \\
\mathbf{V}=\frac{\mathbf{I}+\mathbf{J}+\mathbf{G}+\mathbf{E}+\mathbf{H}+\mathbf{F}}{6} \\
\mathbf{O}=r \mathbf{I}+r \mathbf{J}+r \mathbf{H}+(1-3 r) \mathbf{A} \\
\mathbf{U}=t \mathbf{I}+t \mathbf{J}+(1-2 t) \mathbf{A}
\end{array}\right.
$$

where the specification of $O$ and $U$ are also included for completeness. These four points always 
form a quadrilateral according to the following lemma.

Lemma 2 The four points, $W, V, O$, and $U$ as defined in (6), are always coplanar.

Proof: According to the distribution of the input points, there exists $\lambda, \eta$ such that

$$
\left\{\begin{array}{lll}
\mathbf{I}=\lambda \mathbf{A}+(1-\lambda) \mathbf{B}, & & \mathbf{G}=\eta(\mathbf{B}+\mathbf{C})+(1-2 \eta) \mathbf{A} \\
\mathbf{J}=\lambda \mathbf{A}+(1-\lambda) \mathbf{C}, & & \mathbf{E}=\eta(\mathbf{C}+\mathbf{D})+(1-2 \eta) \mathbf{A} \\
\mathbf{H}=\lambda \mathbf{A}+(1-\lambda) \mathbf{D}, & & \mathbf{F}=\eta(\mathbf{B}+\mathbf{D})+(1-2 \eta) \mathbf{A}
\end{array}\right.
$$

Substitute the above formula into (6) to obtain

$$
\left(\begin{array}{llll}
\mathbf{U} & \mathbf{O} & \mathbf{W} & \mathbf{V}
\end{array}\right)=\left(\begin{array}{llll}
\mathbf{A} & \mathbf{B} & \mathbf{C} & \mathbf{D}
\end{array}\right)\left(\begin{array}{cccc}
1-2 t+2 t \lambda & 1-3 r+3 r \lambda & \frac{1-2 \eta+2 \lambda}{3} & \frac{3+3 \lambda-6 \eta}{6} \\
t(1-\lambda) & r(1-\lambda) & \frac{1+\eta-\lambda}{3} & \frac{1-\lambda+2 \eta}{6} \\
t(1-\lambda) & r(1-\lambda) & \frac{1+\eta-\lambda}{3} & \frac{1-\lambda+2 \eta}{6} \\
0 & r(1-\lambda) & 0 & \frac{1-\lambda+2 \eta}{6}
\end{array}\right) .
$$

The matrix in the above is singular, which proves the lemma.

Remark 1 For the points which are used to compute the second order partition in this section, one have $\lambda=1 / 2, \eta=1 / 3$. These special values for $\lambda$ and $\eta$ are not used in the proof. So the lemma also applies to certain faces of the third and fourth-order partitions which are generated with the algorithm in this section.

\subsection{The Third Order Partition}

To compute the third order partition, we choose the interpolation points from [6]. All these points are on the surface of the tetrahedron. In the upper left part of Fig. 3, we plot the points which are on one tetrahedron face. Using the algorithm to compute the second order partition, a sub-cell can be constructed for all the points shown in the picture except $P$. For example, when building sub-cells for the three points on the top of the picture, we ignore all the interpolation points on the lowest layer (i.e., the ten points on the bottom tetrahedron face). Then the number of remaining points will be ten, which is exactly the number of second order interpolation points. Treat these ten points as the input points and use the algorithm in Sec. 2.2 to construct the sub-cells for the three top points shown in the picture. Similar procedure can be done for all other points except $P$. 

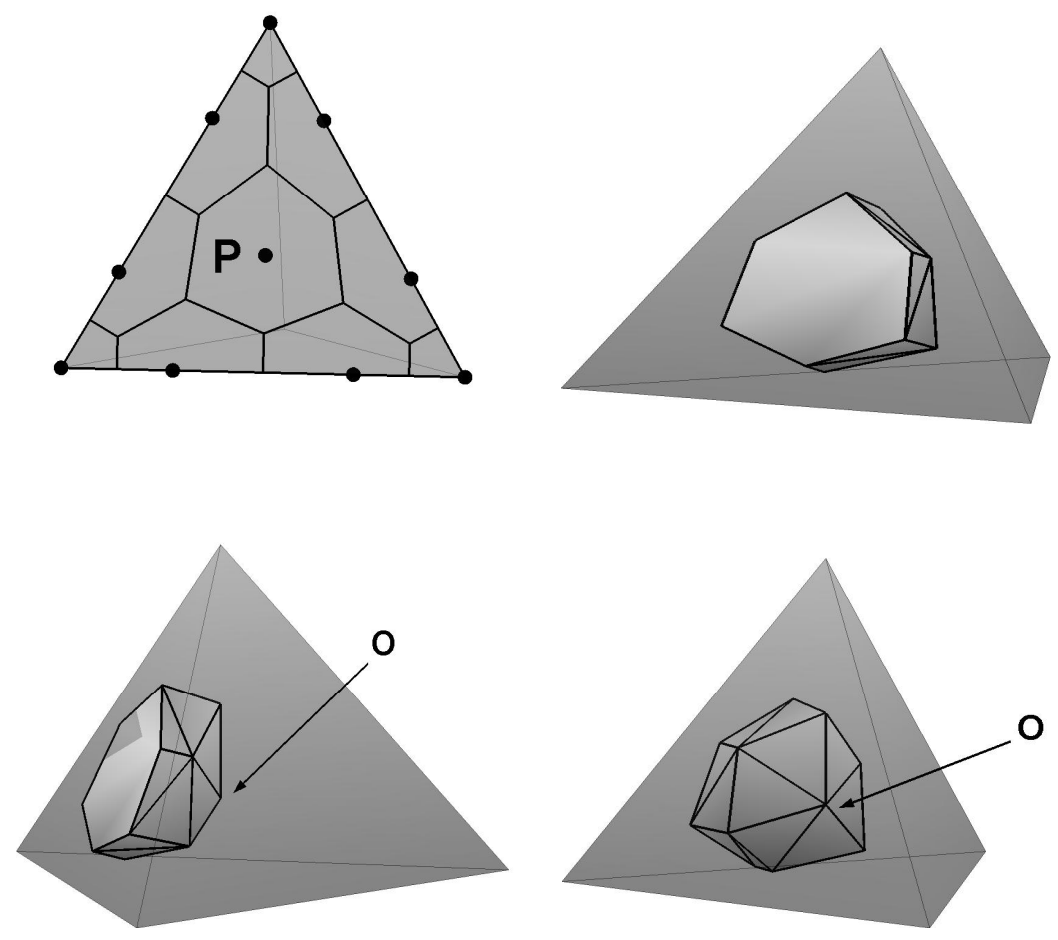

Figure 3: The third order partition of the tetrahedron: a sub-cell.

So the only additional work is to build a sub-cell containing point $P$ for each tetrahedron face. We compute these four sub-cells as follows. Observing that a single connected volume will be left after excluding those sub-cells which have already been built, we choose the mass center of the original tetrahedron as a new vertex, and then connect this new vertex to certain vertices of the single volume to divide it into four identical sub-cells. One of such sub-cell is shown in Fig. 3. In the upper right, it shows a view from the side with point $P$. In the lower left, it is a view after rotating the tetrahedron from right to left for a small angle, where point $O$ is the mass center of the tetrahedron. Finally in the lower right, we rotate it a little more to show the sub-cell from the opposite side. This sub-cell has one hexagonal face and 18 triangular faces. Till now we have computed a third order partition of the tetrahedron. In Sec. 3.3, we will optimize the partition by moving the input points around. 


\subsection{The Fourth Order Partition}

The fourth order interpolation points set [6] has 35 points, only one of which is inside the tetrahedron. Figure 4 shows the points which are on one face of the tetrahedron. For each surface point, a sub-cell can be constructed with the algorithm for the third-order partition. For example, for the six points shown in the top left of Fig. 4, we can simply apply the algorithm for the third-order partition on the points set which includes all the original interpolation points except those on the bottom tetrahedron face. So it seems that we only need a new technique to construct a sub-cell for the interior point. However, even this new technique is not necessary because after building all the other sub-cells, there will be left a single connected volume which is just the sub-cell for the interior point. Figure 4 shows this sub-cell from different perspective. On the top right, it is a view from the reader's side. The bottom left shows a view after rotating the tetrahedron from right to left for a certain angle. The bottom right is another view after further rotation. This sub-cell has 24 triangular faces. The data for the optimized version of the partition will be given in Sec. 3.3.

Remark 2 The recursive algorithm can be used to compute higher order partitions of the tetrahedron. 

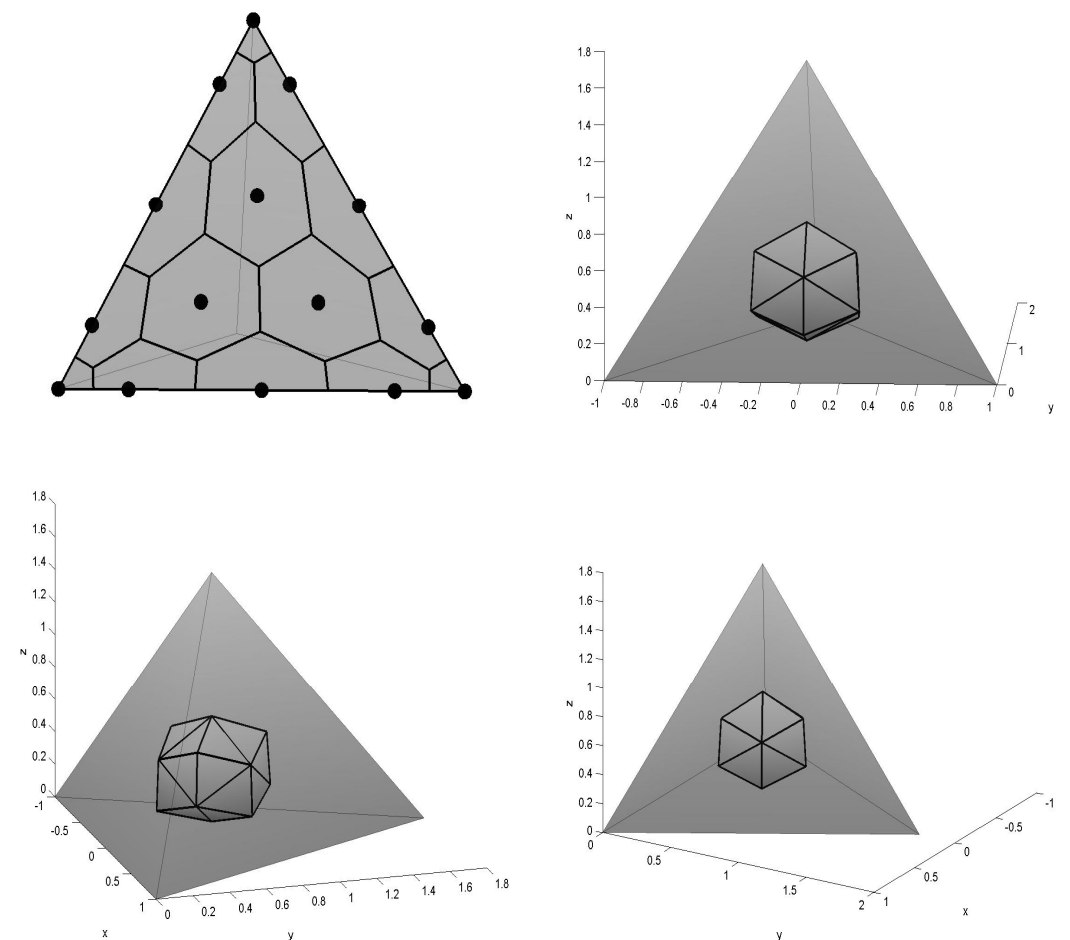

Figure 4: The interior sub-cell of the fourth order partition of the tetrahedron. 


\section{Partition Optimization}

In this section, we optimize the partitions given in [3] and the three-dimensional partitions computed in Sec.2.

\subsection{One Dimensional Partitions}

In [3], we developed several one-dimensional partitions from the Chebyshev and Legendre GaussLobatto points by using middle points of two neighboring input points as partition vertices. However, those partitions have larger Lebesgue constants than the partitions given in [9].

So in this paper, instead of simply using middle points of two neighboring input points as endpoints of sub-cells, we construct a partition in which most input points sit at the center of the sub-cells and the sub-cells have increasing size from the boundary to the interior. In specific, consider an even order partition with input points $-1=x_{0}<x_{1}<\cdots<x_{2 n}=1$. Take $d_{i}=x_{i}-x_{i-1}, i=1, \cdots, 2 n$. Assume $r$ be the portion of interval $\left[x_{n-1}, x_{n}\right]$ that is allocated to the sub-cell including $x_{n}$. So the $(1-r) d_{n}$ of $\left[x_{n-1}, x_{n}\right]$ is allocated to the sub-cell $x_{n-1}$, i.e., $y_{n}=x_{n-1}+(1-r) d_{n}$ is a vertex of the partition. In order to make $x_{n-1}$ be the center of a sub-cell, $y_{n-1}=x_{n-1}-(1-r) d_{n}$ should also be a vertex of the partition. Hence $(1-r) d_{n}$ of the interval $\left[x_{n-2}, x_{n-1}\right]$ is allocated to the sub-cell including $x_{n-1}$. Since we require the sub-cells closer to the middle have larger size, one can have

$$
\left\{\begin{array}{l}
r d_{n} \geq(1-r) d_{n} \\
(1-r) d_{n} \geq \frac{d_{n-1}}{2}
\end{array} \quad \Longrightarrow \quad 1-\frac{d_{n-1}}{2 d_{n}} \geq r \geq \frac{1}{2}\right.
$$

Continue the above process until we get $y_{0}$ such that $x_{0}$ is the middle point of $\left[y_{0}, y_{1}\right]$. Repeat it for the other half interpolation points, $\left\{x_{n+1}, \cdots, x_{2 n}\right\}$, to get the whole partition. Note that the partition will cover a region larger than the original domain $[-1,1]$. A partition of $[-1,1]$ can be easily obtained by a linear mapping. Similar procedure can be done for odd order partitions.

In the algorithm, $r$ is the only parameter to be optimized. With the above algorithm, we compute the optimal partitions from the Legendre-Gauss-Lobatto, Chebyshev-Gauss-Lobatto points, and the optimal polynomial interpolation set [1]. These optimized partitions are denoted as $\Pi_{L G L}$, 
$\Pi_{C G L}$, and $\Pi_{T_{1}}$ respectively, among which $\Pi_{L G L}$ is the smallest for partitions of most order. The results are shown in Tab. 4-5.

Table 4: Lebesgue constants for several one-dimensional partitions.

\begin{tabular}{ccccccc}
\hline order & $\Pi_{L G L}$ & $\mathrm{r}$ & $\Pi_{C G L}$ & $\mathrm{r}$ & $\Pi_{T_{1}}$ & $\mathrm{r}$ \\
\hline 2 & 1.685 & 0.877 & 1.685 & 0.877 & 1.685 & 0.877 \\
3 & 1.823 & 0.884 & 1.823 & 0.872 & 1.823 & 0.890 \\
4 & 1.950 & 0.533 & 2.132 & 0.656 & 2.674 & 0.500 \\
5 & 2.108 & 0.562 & 2.416 & 0.542 & 2.337 & 0.500 \\
6 & 2.291 & 0.542 & 2.560 & 0.500 & 2.241 & 0.593 \\
7 & 2.449 & 0.554 & 2.666 & 0.500 & 2.387 & 0.602 \\
8 & 2.545 & 0.514 & 2.834 & 0.571 & 3.898 & 0.500 \\
9 & 2.644 & 0.521 & 3.015 & 0.518 & 3.514 & 0.500 \\
10 & 2.748 & 0.512 & 3.105 & 0.500 & 3.190 & 0.541 \\
11 & 2.840 & 0.519 & 3.180 & 0.500 & 2.920 & 0.552 \\
12 & 2.918 & 0.509 & 3.268 & 0.534 & 4.711 & 0.500 \\
13 & 2.991 & 0.511 & 3.384 & 0.509 & 4.343 & 0.500 \\
14 & 3.063 & 0.504 & 3.450 & 0.500 & 4.020 & 0.522 \\
15 & 3.124 & 0.507 & 3.509 & 0.500 & 3.738 & 0.529 \\
16 & 3.190 & 0.507 & 3.571 & 0.519 & 5.312 & 0.500 \\
17 & 3.248 & 0.507 & 3.653 & 0.505 & 4.969 & 0.500 \\
18 & 3.303 & 0.500 & 3.705 & 0.500 & 4.661 & 0.513 \\
19 & 3.354 & 0.505 & 3.753 & 0.500 & 4.384 & 0.519 \\
20 & 3.404 & 0.507 & 3.801 & 0.512 & & \\
\hline
\end{tabular}

Table 5: Node sets of the partitions $\Pi_{L G L}$. Only positive interior points are listed.

\begin{tabular}{cc}
\hline order & $x_{i}$ \\
\hline 2 & 0.7812757765 \\
3 & 0.8797040574 \\
4 & 0.3358143267 \\
& 0.9232452092 \\
5 & 0.5414344225 \\
& 0.9518023783 \\
6 & 0.2485522653 \\
& 0.6680769262 \\
& 0.9550635604 \\
7 & 0.4131856053 \\
& 0.7482430030 \\
& 0.9628667435 \\
8 & 0.1837943613 \\
& 0.5315587191 \\
& 0.8025200275 \\
\hline
\end{tabular}




\begin{tabular}{cc}
\multicolumn{2}{c}{ Table 5 (Contd.) } \\
\hline order & $x_{i}$ \\
\hline \multirow{3}{*}{9} & 0.9700321565 \\
& 0.3247408153 \\
& 0.6207881103 \\
& 0.8408055127 \\
& 0.9784046134 \\
10 & 0.1498237400 \\
& 0.4354893164 \\
& 0.6831261231 \\
& 0.8693871112 \\
& 0.9790260552 \\
\hline
\end{tabular}

\subsection{Two Dimensional Partitions}

We only optimize the partitions of up to the fourth order in the triangle which are given in [3]. Different constraint will be applied when optimizing partitions of different order.

\subsubsection{The Second Order Partition of the Triangle}

Figure 5 shows the second order partition from [3]. We seek an optimal partition whose vertices on each triangle edge are just the vertices of the one-dimensional second-order partition computed in Sec. 3.1. So only three vertices of the partition, points $E, F, G$, are not fixed. Because of symmetry, points $E, F, G$ are determined by a single parameter. Specially, their barycenter coordinates can be expressed as $(r, r, 1-2 r),(r, 1-2 r, r),(1-2 r, r, r)$. Under the above constraint, the optimal partition (Fig. 6) has Lebesgue constant 3.0630, and the partition data is listed in Tab. 6-7. We also tried to move around the partition vertices which are on the triangle edges. There is little difference in the optimal Lebesgue constant.

Table 6: The second order partition of the triangle: the barycenter coordinates of the vertices.

\begin{tabular}{cccc}
\hline$i$ & & & \\
\hline 1 & 1.0000000000 & 0.0000000000 & 0.0000000000 \\
2 & 0.8906378883 & 0.1093621117 & 0.0000000000 \\
3 & 0.8269977508 & 0.0865011246 & 0.0865011246 \\
4 & 0.8906378883 & 0.0000000000 & 0.1093621117 \\
5 & 0.1093621117 & 0.8906378883 & 0.0000000000 \\
6 & 0.0865011246 & 0.8269977508 & 0.0865011246 \\
7 & 0.3333333334 & 0.3333333334 & 0.3333333331 \\
\hline
\end{tabular}


Table 6 (Contd.)

\begin{tabular}{cccc}
\hline$i$ & & & \\
\hline 8 & 0.0865011246 & 0.0865011246 & 0.8269977508 \\
9 & 0.1093621117 & 0.0000000000 & 0.8906378883 \\
10 & 0.0000000000 & 1.0000000000 & 0.0000000000 \\
11 & 0.0000000000 & 0.8906378883 & 0.1093621117 \\
12 & 0.0000000000 & 0.1093621117 & 0.8906378883 \\
13 & 0.0000000000 & 0.0000000000 & 1.0000000000 \\
\hline
\end{tabular}

Table 7: The second order partition of the triangle: the sub-cells. Each row lists the indices of vertices in counterclockwise direction for one sub-cell. $M$ denotes the number of vertices of each individual face.

\begin{tabular}{cc|ccccc}
\hline$i$ & $M$ & & & & & \\
\hline 1 & 4 & 9 & 8 & 12 & 13 & \\
2 & 5 & 4 & 3 & 7 & 8 & 9 \\
3 & 5 & 8 & 7 & 6 & 11 & 12 \\
4 & 4 & 1 & 2 & 3 & 4 & \\
5 & 5 & 3 & 2 & 5 & 6 & 7 \\
6 & 4 & 6 & 5 & 10 & 11 & \\
\hline
\end{tabular}

\subsubsection{The Third Order Partition of the Triangle}

In [3], we propose a technique to compute the partitions from the layering structure of the input points. In that algorithm, we first generate a triangularization from the input points. The centroids of the triangles in the triangularization are then used as the vertices of the partition. (See [3] for further details.) Here we apply the same algorithm to compute the third order partition except that a different point instead of the centroid in each triangle is chosen to be a vertex of the partition. In particular, we choose a weighted average of the triangle vertices, with the weight being the largest barycenter coordinate of each triangle vertex. Within the above algorithm, we move around the input points while keeping its symmetry property to obtain a partition with the smallest Lebesgue constant. The optimal partition obtained this way is shown in Fig. 6, and its Lebesgue constant is 3.2129. The partition data is given in Tab.8-9. We also tried to compute the optimal partition under the constraint similar to Sec. 3.2.1. The result is not as good as the one we give here. 


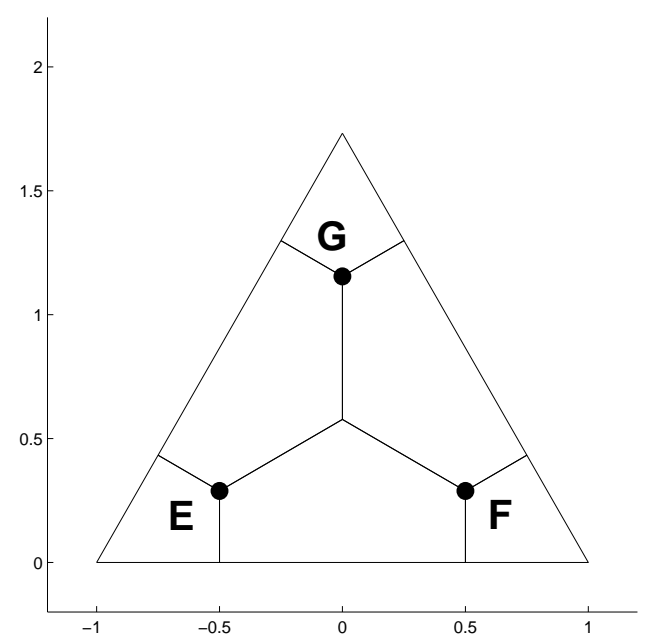

Figure 5: The second order partition of the triangle.

Table 8: The third order partition of the triangle: the barycenter coordinates of the vertices.

\begin{tabular}{cccc}
\hline$i$ & & & \\
\hline 1 & 0.9574919119 & 0.0212540441 & 0.0212540441 \\
2 & 0.4747800545 & 0.4747800545 & 0.0504398910 \\
3 & 0.8437475098 & 0.0781262451 & 0.0781262451 \\
4 & 0.4747800545 & 0.0504398911 & 0.4747800544 \\
5 & 0.0212540441 & 0.9574919119 & 0.0212540441 \\
6 & 0.0781262451 & 0.8437475098 & 0.0781262451 \\
7 & 0.0504398911 & 0.4747800545 & 0.4747800544 \\
8 & 0.0781262451 & 0.0781262451 & 0.8437475097 \\
9 & 0.0212540441 & 0.0212540441 & 0.9574919119 \\
10 & 1.0000000000 & 0.0000000000 & 0.0000000000 \\
11 & 0.0000000000 & 0.0000000000 & 1.0000000000 \\
12 & 0.0000000000 & 1.0000000000 & 0.0000000000 \\
13 & 0.9673771699 & 0.0326228301 & 0.0000000000 \\
14 & 0.5000000000 & 0.5000000000 & 0.0000000000 \\
15 & 0.0326228301 & 0.9673771699 & 0.0000000000 \\
16 & 0.9673771699 & 0.0000000000 & 0.0326228301 \\
17 & 0.5000000000 & 0.0000000000 & 0.5000000000 \\
18 & 0.0326228301 & 0.0000000000 & 0.9673771699 \\
19 & 0.0000000000 & 0.9673771699 & 0.0326228301 \\
20 & 0.0000000000 & 0.5000000000 & 0.5000000000 \\
21 & 0.0000000000 & 0.0326228301 & 0.9673771699 \\
\hline
\end{tabular}

Table 9: The third order partition of the triangle: the sub-cells.

\begin{tabular}{cc|cccccc}
\hline$i$ & $M$ & & & & & & \\
\hline 1 & 4 & 10 & 13 & 1 & 16 & & \\
2 & 5 & 1 & 13 & 14 & 2 & 3 & \\
3 & 5 & 16 & 118 & 3 & 4 & 17 & \\
4 & 5 & 2 & 14 & 15 & 5 & 6 & \\
5 & 6 & 4 & 3 & 2 & 6 & 7 & 8 \\
6 & 5 & 17 & 4 & 8 & 9 & 18 &
\end{tabular}



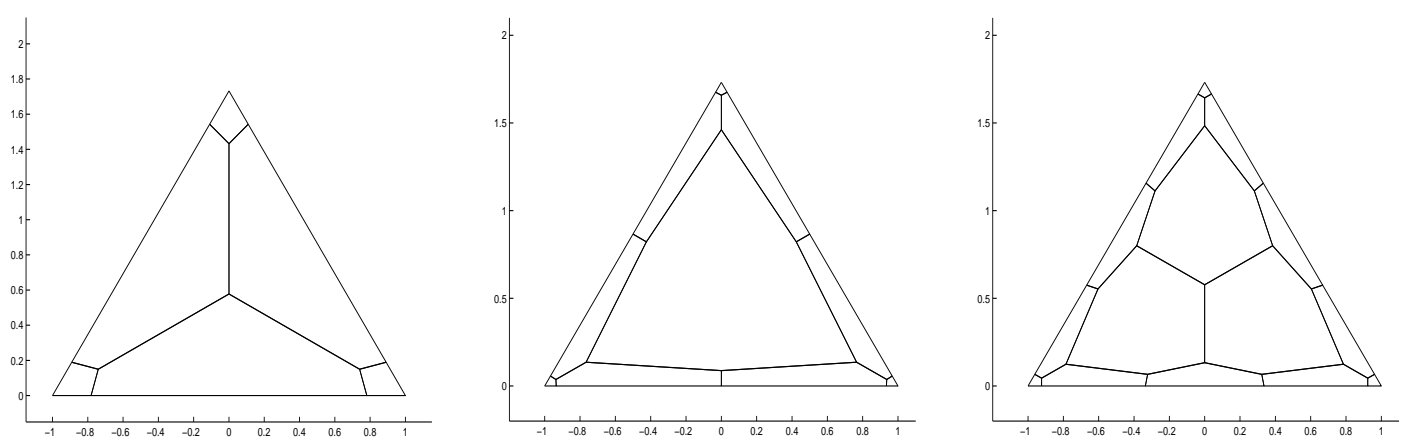

Figure 6: Several two-dimensional partitions. Left: second order; Middle: third order; Right: fourth order.

partition vertices to get a small Lebesgue constant. The partition shown in Fig. 6 has Lebesgue constant 4.0563. Note that this partition is not optimal. But when perturbing the partition vertices, only a few percent change is observed in the Lebesgue constant. The partition data is given in Tab. $10-11$.

Table 10: The fourth order partition of the triangle: the barycenter coordinates of the vertices.

\begin{tabular}{cccc}
\hline$i$ & & & \\
\hline 1 & 1.0000000000 & 0.0000000000 & 0.0000000000 \\
2 & 0.9616226046 & 0.0383773954 & 0.0000000000 \\
3 & 0.9488301395 & 0.0255849303 & 0.0255849303 \\
4 & 0.9616226046 & 0.0000000000 & 0.0383773954 \\
5 & 0.6679071634 & 0.3320928367 & 0.0000000000 \\
6 & 0.6422746261 & 0.3193479785 & 0.0383773954 \\
7 & 0.8568233902 & 0.0715883049 & 0.0715883049 \\
8 & 0.6422746261 & 0.0383773954 & 0.3193479785 \\
9 & 0.6679071634 & 0.0000000000 & 0.3320928367 \\
10 & 0.3320928367 & 0.6679071634 & 0.0000000000 \\
11 & 0.3193479785 & 0.6422746261 & 0.0383773954 \\
12 & 0.4616226046 & 0.4616226046 & 0.0767547908 \\
13 & 0.3333333334 & 0.3333333334 & 0.3333333331 \\
14 & 0.4616226046 & 0.0767547908 & 0.4616226046 \\
15 & 0.3193479785 & 0.0383773954 & 0.6422746261 \\
16 & 0.3320928367 & 0.0000000000 & 0.6679071634 \\
17 & 0.0383773954 & 0.9616226046 & 0.0000000000 \\
18 & 0.0255849303 & 0.9488301395 & 0.0255849303 \\
19 & 0.0715883049 & 0.8568233902 & 0.0715883049 \\
20 & 0.0383773954 & 0.6422746261 & 0.3193479785 \\
21 & 0.0767547908 & 0.4616226046 & 0.4616226046 \\
22 & 0.0383773954 & 0.3193479785 & 0.6422746261 \\
23 & 0.0715883049 & 0.0715883049 & 0.8568233902 \\
\hline & & &
\end{tabular}


Table 10 (Contd.)

\begin{tabular}{cccc}
\hline$i$ & & & \\
\hline 24 & 0.0255849303 & 0.0255849303 & 0.9488301395 \\
25 & 0.0383773954 & 0.0000000000 & 0.9616226046 \\
26 & 0.0000000000 & 1.0000000000 & 0.0000000000 \\
27 & 0.0000000000 & 0.9616226046 & 0.0383773954 \\
28 & 0.0000000000 & 0.6679071634 & 0.3320928367 \\
29 & 0.0000000000 & 0.3320928367 & 0.6679071634 \\
30 & 0.0000000000 & 0.0383773954 & 0.9616226046 \\
31 & 0.0000000000 & 0.0000000000 & 1.0000000000 \\
\hline
\end{tabular}

Table 11: The fourth order partition of the triangle: the sub-cells.

\begin{tabular}{cc|cccccc}
\hline$i$ & $M$ & & & & & \\
\hline 1 & 4 & 25 & 24 & 30 & 31 & & \\
2 & 5 & 16 & 15 & 23 & 24 & 25 & \\
3 & 5 & 24 & 23 & 22 & 29 & 30 & \\
4 & 5 & 9 & 8 & 14 & 15 & 16 & \\
5 & 6 & 14 & 13 & 21 & 22 & 23 & 15 \\
6 & 5 & 22 & 21 & 20 & 28 & 29 & \\
7 & 5 & 4 & 3 & 7 & 8 & 9 & \\
8 & 6 & 7 & 6 & 12 & 13 & 14 & 8 \\
9 & 6 & 13 & 12 & 11 & 19 & 20 & 21 \\
10 & 5 & 20 & 19 & 18 & 27 & 28 & \\
11 & 4 & 1 & 2 & 3 & 4 & & \\
12 & 5 & 2 & 5 & 6 & 7 & 3 & \\
13 & 5 & 6 & 5 & 10 & 11 & 12 & \\
14 & 5 & 10 & 17 & 18 & 19 & 11 & \\
15 & 4 & 18 & 17 & 26 & 27 & & \\
\hline
\end{tabular}

\subsection{Three Dimensional Partitions}

For the second order partition, we use the optimized second order partition of the triangle computed in Sec. 3.2.1 on the faces of the tetrahedron. In order to minimize the number of total faces, the partition vertices inside the tetrahedron are chosen such that the condition of Lemma 1 is satisfied. Then the partition is actually fixed, which is shown in Fig. 7. The partition has 37 vertices and 48 faces, and its Lebesgue constant is 5.0814. The partition data is listed in Tab. 12-14.

For the third and fourth order partitions, we simple employ the algorithm given in Sec. 2.3 and 2.4 to compute the partition, and try to find the one with minimal Lebesgue constant by moving 


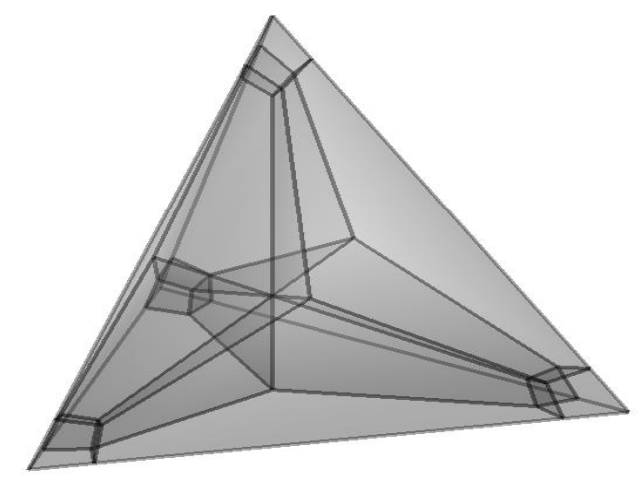

Figure 7: Second order partition of tetrahedron.

the input points around. The smallest Lebesgue constants we found for the third and fourth order partitions are 6.8725 and 7.9940 respectively. The obtained third order partition has 73 vertices and 130 faces, while the fourth order partition has 126 vertices and 252 faces. The partition data is listed in Tab. 15-20. Note that this fourth order partition has some non-convex sub-cells.

Table 12: The second order partition of the tetrahedron: the barycenter coordinates of the vertices.

\begin{tabular}{ccccc}
\hline$i$ & & & & \\
\hline 1 & 0.0000000000 & 1.0000000000 & 0.0000000000 & 0.0000000000 \\
2 & 0.0000000000 & 0.8906378883 & 0.1093621117 & 0.0000000000 \\
3 & 0.0000000000 & 0.8906378883 & 0.0000000000 & 0.1093621117 \\
4 & 0.0000000000 & 0.8269977508 & 0.0865011246 & 0.0865011246 \\
5 & 0.0000000000 & 0.0000000000 & 1.0000000000 & 0.0000000000 \\
6 & 0.0000000000 & 0.1093621117 & 0.8906378883 & 0.0000000000 \\
7 & 0.0000000000 & 0.0000000000 & 0.8906378883 & 0.1093621117 \\
8 & 0.0000000000 & 0.0865011246 & 0.8269977508 & 0.0865011246 \\
9 & 0.0000000000 & 0.0000000000 & 0.0000000000 & 1.0000000000 \\
10 & 0.0000000000 & 0.1093621117 & 0.0000000000 & 0.8906378883 \\
11 & 0.0000000000 & 0.0000000000 & 0.1093621117 & 0.8906378883 \\
12 & 0.0000000000 & 0.0865011246 & 0.0865011246 & 0.8269977508 \\
13 & 0.0000000000 & 0.3333333334 & 0.3333333334 & 0.3333333331 \\
14 & 0.0715453331 & 0.7853640006 & 0.0715453331 & 0.0715453331 \\
15 & 0.0715453331 & 0.0715453331 & 0.7853640006 & 0.0715453331 \\
16 & 0.0715453331 & 0.0715453331 & 0.0715453331 & 0.7853640006 \\
17 & 0.0865011246 & 0.8269977508 & 0.0865011246 & 0.0000000000 \\
18 & 0.0865011246 & 0.8269977508 & 0.0000000000 & 0.0865011246 \\
19 & 0.0865011246 & 0.0865011246 & 0.8269977508 & 0.0000000000 \\
\hline
\end{tabular}


Table 12 (Contd.)

\begin{tabular}{ccccc}
\hline$i$ & & & & \\
\hline 20 & 0.0865011246 & 0.0000000000 & 0.8269977508 & 0.0865011246 \\
21 & 0.0865011246 & 0.0000000000 & 0.0865011246 & 0.8269977508 \\
22 & 0.0865011246 & 0.0865011246 & 0.0000000000 & 0.8269977508 \\
23 & 0.1093621117 & 0.8906378883 & 0.0000000000 & 0.0000000000 \\
24 & 0.1093621117 & 0.0000000000 & 0.8906378883 & 0.0000000000 \\
25 & 0.1093621117 & 0.0000000000 & 0.0000000000 & 0.8906378883 \\
26 & 0.2500000000 & 0.2500000000 & 0.2500000000 & 0.2500000000 \\
27 & 0.3333333334 & 0.3333333334 & 0.3333333331 & 0.0000000000 \\
28 & 0.3333333334 & 0.3333333334 & 0.0000000000 & 0.3333333331 \\
29 & 0.3333333334 & 0.0000000000 & 0.3333333334 & 0.3333333331 \\
30 & 0.7853640006 & 0.0715453331 & 0.0715453331 & 0.0715453331 \\
31 & 0.8269977508 & 0.0865011246 & 0.0865011246 & 0.0000000000 \\
32 & 0.8269977508 & 0.0000000000 & 0.0865011246 & 0.0865011246 \\
33 & 0.8269977508 & 0.0865011246 & 0.0000000000 & 0.0865011246 \\
34 & 0.8906378883 & 0.1093621117 & 0.0000000000 & 0.0000000000 \\
35 & 0.8906378883 & 0.0000000000 & 0.1093621117 & 0.0000000000 \\
36 & 0.8906378883 & 0.0000000000 & 0.0000000000 & 0.1093621117 \\
37 & 1.0000000000 & 0.0000000000 & 0.0000000000 & 0.0000000000 \\
\hline
\end{tabular}

Table 13: The second order partition of the tetrahedron: the faces. Each row lists the indices of vertices in counterclockwise order for one face. $M$ denotes the number of vertices of each individual face.

\begin{tabular}{cc|cccc}
\hline$i$ & $M$ & & & & \\
\hline 1 & 4 & 31 & 35 & 37 & 34 \\
2 & 4 & 32 & 36 & 37 & 35 \\
3 & 4 & 33 & 36 & 37 & 34 \\
4 & 4 & 30 & 33 & 36 & 32 \\
5 & 4 & 30 & 33 & 34 & 31 \\
6 & 4 & 30 & 32 & 35 & 31 \\
7 & 4 & 1 & 23 & 17 & 2 \\
8 & 4 & 1 & 3 & 4 & 2 \\
9 & 4 & 1 & 23 & 18 & 3 \\
10 & 4 & 3 & 18 & 14 & 4 \\
11 & 4 & 14 & 18 & 23 & 17 \\
12 & 4 & 2 & 17 & 14 & 4 \\
13 & 4 & 5 & 24 & 19 & 6 \\
14 & 4 & 5 & 24 & 20 & 7 \\
15 & 4 & 5 & 7 & 8 & 6 \\
16 & 4 & 7 & 20 & 15 & 8 \\
17 & 4 & 6 & 19 & 15 & 8 \\
18 & 4 & 15 & 20 & 24 & 19 \\
19 & 4 & 9 & 11 & 12 & 10 \\
\hline
\end{tabular}


Table 13 (Contd.)

\begin{tabular}{cc|ccccc}
\hline$i$ & $M$ & & & & & \\
\hline 20 & 4 & 9 & 25 & 21 & 11 & \\
21 & 4 & 9 & 25 & 22 & 10 & \\
22 & 4 & 16 & 22 & 25 & 21 & \\
23 & 4 & 10 & 22 & 16 & 12 & \\
24 & 4 & 11 & 21 & 16 & 12 & \\
25 & 5 & 17 & 27 & 31 & 34 & 23 \\
26 & 5 & 18 & 28 & 33 & 34 & 23 \\
27 & 4 & 26 & 30 & 31 & 27 & \\
28 & 4 & 26 & 30 & 33 & 28 & \\
29 & 4 & 14 & 26 & 27 & 17 & \\
30 & 4 & 14 & 26 & 28 & 18 & \\
31 & 5 & 19 & 27 & 31 & 35 & 24 \\
32 & 5 & 20 & 29 & 32 & 35 & 24 \\
33 & 4 & 26 & 30 & 32 & 29 & \\
34 & 4 & 15 & 26 & 27 & 19 & \\
35 & 4 & 15 & 26 & 29 & 20 & \\
36 & 5 & 22 & 28 & 33 & 36 & 25 \\
37 & 5 & 21 & 29 & 32 & 36 & 25 \\
38 & 4 & 16 & 26 & 28 & 22 & \\
39 & 4 & 16 & 26 & 29 & 21 & \\
40 & 5 & 2 & 6 & 8 & 13 & 4 \\
41 & 5 & 2 & 17 & 27 & 19 & 6 \\
42 & 4 & 4 & 14 & 26 & 13 & \\
43 & 4 & 8 & 15 & 26 & 13 & \\
44 & 5 & 3 & 10 & 12 & 13 & 4 \\
45 & 5 & 3 & 18 & 28 & 22 & 10 \\
46 & 4 & 12 & 16 & 26 & 13 & \\
47 & 5 & 7 & 11 & 12 & 13 & 8 \\
48 & 5 & 7 & 20 & 29 & 21 & 11 \\
\hline & & & & & &
\end{tabular}

Table 14: The second order partition of the tetrahedron: the sub-cells. Each row lists the indices of the faces of one sub-cell. $M$ denotes the number of faces of each individual sub-cell.

\begin{tabular}{cc|cccccccc}
\hline$i$ & $M$ & \multicolumn{1}{|c}{} & \multicolumn{1}{|c}{} & & & & & & \\
\hline 1 & 6 & 1 & 2 & 3 & 4 & 5 & 6 & & \\
2 & 6 & 7 & 8 & 9 & 10 & 11 & 12 & & \\
3 & 6 & 13 & 14 & 15 & 16 & 17 & 18 & & \\
4 & 6 & 19 & 20 & 21 & 22 & 23 & 24 & & \\
5 & 8 & 25 & 26 & 5 & 11 & 27 & 28 & 29 & 30 \\
6 & 8 & 31 & 32 & 6 & 18 & 27 & 33 & 34 & 35 \\
7 & 8 & 36 & 37 & 4 & 22 & 28 & 33 & 38 & 39 \\
8 & 8 & 40 & 41 & 12 & 17 & 42 & 29 & 43 & 34 \\
9 & 8 & 44 & 45 & 10 & 23 & 42 & 30 & 46 & 38 \\
\hline
\end{tabular}


Table 14 (Contd.)

\begin{tabular}{cc|cccccccc}
\hline$i$ & $M$ & & & & & & & & \\
\hline 10 & 8 & 47 & 48 & 16 & 24 & 43 & 35 & 46 & 39 \\
\hline
\end{tabular}

Table 15: The third order partition of the tetrahedron: the barycenter coordinates of the vertices.

\begin{tabular}{ccccc}
\hline$i$ & & & & \\
\hline 1 & 0.0000000000 & 1.0000000000 & 0.0000000000 & 0.0000000000 \\
2 & 0.0000000000 & 0.9750000000 & 0.0250000000 & 0.0000000000 \\
3 & 0.0000000000 & 0.9750000000 & 0.0000000000 & 0.0250000000 \\
4 & 0.0000000000 & 0.9666666667 & 0.0166666667 & 0.0166666667 \\
5 & 0.0000000000 & 0.0000000000 & 1.0000000000 & 0.0000000000 \\
6 & 0.0000000000 & 0.0000000000 & 0.9750000000 & 0.0250000000 \\
7 & 0.0000000000 & 0.0250000000 & 0.9750000000 & 0.0000000000 \\
8 & 0.0000000000 & 0.0166666667 & 0.9666666667 & 0.0166666667 \\
9 & 0.0000000000 & 0.0000000000 & 0.0000000000 & 1.0000000000 \\
10 & 0.0000000000 & 0.0250000000 & 0.0000000000 & 0.9750000000 \\
11 & 0.0000000000 & 0.0000000000 & 0.0250000000 & 0.9750000000 \\
12 & 0.0000000000 & 0.0166666667 & 0.0166666667 & 0.9666666667 \\
13 & 0.0000000000 & 0.5000000000 & 0.5000000000 & 0.0000000000 \\
14 & 0.0000000000 & 0.4444444444 & 0.4444444444 & 0.111111111 \\
15 & 0.0000000000 & 0.7444444444 & 0.1277777778 & 0.1277777778 \\
16 & 0.0000000000 & 0.0000000000 & 0.5000000000 & 0.5000000000 \\
17 & 0.0000000000 & 0.11111111 & 0.4444444444 & 0.4444444444 \\
18 & 0.0000000000 & 0.1277777778 & 0.7444444444 & 0.1277777778 \\
19 & 0.0000000000 & 0.5000000000 & 0.0000000000 & 0.5000000000 \\
20 & 0.0000000000 & 0.4444444444 & 0.111111111 & 0.4444444444 \\
21 & 0.0000000000 & 0.1277777778 & 0.1277777778 & 0.7444444444 \\
22 & 0.0125000000 & 0.9625000000 & 0.0125000000 & 0.0125000000 \\
23 & 0.0125000000 & 0.0125000000 & 0.9625000000 & 0.0125000000 \\
24 & 0.0125000000 & 0.0125000000 & 0.0125000000 & 0.9625000000 \\
25 & 0.0166666667 & 0.9666666667 & 0.0000000000 & 0.0166666667 \\
26 & 0.0166666667 & 0.9666666667 & 0.0166666667 & 0.0000000000 \\
27 & 0.0166666667 & 0.0000000000 & 0.9666666667 & 0.0166666667 \\
28 & 0.0166666667 & 0.0166666667 & 0.9666666667 & 0.0000000000 \\
29 & 0.0166666667 & 0.0166666667 & 0.0000000000 & 0.9666666667 \\
30 & 0.0166666667 & 0.0000000000 & 0.0166666667 & 0.9666666667 \\
31 & 0.0250000000 & 0.9750000000 & 0.0000000000 & 0.0000000000 \\
32 & 0.0250000000 & 0.0000000000 & 0.9750000000 & 0.0000000000 \\
33 & 0.0250000000 & 0.0000000000 & 0.0000000000 & 0.9750000000 \\
34 & 0.0833333333 & 0.4166666667 & 0.4166666667 & 0.0833333333 \\
35 & 0.0833333333 & 0.0833333333 & 0.4166666667 & 0.4166666667 \\
36 & 0.0833333333 & 0.4166666667 & 0.0833333333 & 0.4166666667 \\
37 & 0.1111111111 & 0.4444444444 & 0.4444444444 & 0.0000000000 \\
\hline & & & & \\
\hline
\end{tabular}


Table 15 (Contd.)

\begin{tabular}{ccccc}
\hline$i$ & & & & \\
\hline 38 & 0.1111111111 & 0.0000000000 & 0.4444444444 & 0.4444444444 \\
39 & 0.111111111 & 0.4444444444 & 0.0000000000 & 0.4444444444 \\
40 & 0.1194444444 & 0.6416666667 & 0.1194444444 & 0.1194444444 \\
41 & 0.1194444444 & 0.1194444444 & 0.6416666667 & 0.1194444444 \\
42 & 0.1194444444 & 0.1194444444 & 0.1194444444 & 0.6416666667 \\
43 & 0.1277777778 & 0.7444444444 & 0.1277777778 & 0.0000000000 \\
44 & 0.1277777778 & 0.0000000000 & 0.7444444444 & 0.1277777778 \\
45 & 0.1277777778 & 0.1277777778 & 0.0000000000 & 0.7444444444 \\
46 & 0.1277777778 & 0.0000000000 & 0.1277777778 & 0.7444444444 \\
47 & 0.1277777778 & 0.7444444444 & 0.0000000000 & 0.1277777778 \\
48 & 0.1277777778 & 0.1277777778 & 0.7444444444 & 0.0000000000 \\
49 & 0.2500000000 & 0.2500000000 & 0.2500000000 & 0.2500000000 \\
50 & 0.4166666667 & 0.4166666667 & 0.0833333333 & 0.0833333333 \\
51 & 0.4166666667 & 0.0833333333 & 0.0833333333 & 0.4166666667 \\
52 & 0.4166666667 & 0.0833333333 & 0.4166666667 & 0.0833333333 \\
53 & 0.4444444444 & 0.4444444444 & 0.111111111 & 0.0000000000 \\
54 & 0.4444444444 & 0.4444444444 & 0.0000000000 & 0.111111111 \\
55 & 0.4444444444 & 0.111111111 & 0.0000000000 & 0.4444444444 \\
56 & 0.4444444444 & 0.0000000000 & 0.111111111 & 0.4444444444 \\
57 & 0.4444444444 & 0.0000000000 & 0.4444444444 & 0.111111111 \\
58 & 0.4444444444 & 0.111111111 & 0.4444444444 & 0.0000000000 \\
59 & 0.5000000000 & 0.5000000000 & 0.0000000000 & 0.0000000000 \\
60 & 0.5000000000 & 0.0000000000 & 0.0000000000 & 0.5000000000 \\
61 & 0.5000000000 & 0.0000000000 & 0.5000000000 & 0.0000000000 \\
62 & 0.6416666667 & 0.1194444444 & 0.1194444444 & 0.1194444444 \\
63 & 0.7444444444 & 0.1277777778 & 0.1277777778 & 0.0000000000 \\
64 & 0.7444444444 & 0.1277777778 & 0.0000000000 & 0.1277777778 \\
65 & 0.7444444444 & 0.0000000000 & 0.1277777778 & 0.1277777778 \\
66 & 0.9625000000 & 0.0125000000 & 0.0125000000 & 0.0125000000 \\
67 & 0.9666666667 & 0.0166666667 & 0.0166666667 & 0.0000000000 \\
68 & 0.9666666667 & 0.0000000000 & 0.0166666667 & 0.0166666667 \\
69 & 0.9666666667 & 0.0166666667 & 0.0000000000 & 0.0166666667 \\
70 & 0.9750000000 & 0.0250000000 & 0.0000000000 & 0.0000000000 \\
71 & 0.9750000000 & 0.0000000000 & 0.0250000000 & 0.0000000000 \\
72 & 0.9750000000 & 0.0000000000 & 0.0000000000 & 0.0250000000 \\
73 & 1.0000000000 & 0.0000000000 & 0.0000000000 & 0.0000000000 \\
\hline & & & &
\end{tabular}

Table 16: The third order partition of the tetrahedron: the faces.

\begin{tabular}{cc|cccc}
\hline$i$ & $M$ & & & & \\
\hline 1 & 4 & 67 & 71 & 73 & 70 \\
2 & 4 & 68 & 72 & 73 & 71 \\
\hline
\end{tabular}


Table 16 (Contd.)

\begin{tabular}{|c|c|c|c|c|c|c|}
\hline$i$ & $M$ & & & & & \\
\hline 3 & 4 & 69 & 72 & 73 & 70 & \\
\hline 4 & 4 & 66 & 69 & 72 & 68 & \\
\hline 5 & 4 & 66 & 69 & 70 & 67 & \\
\hline 6 & 4 & 66 & 68 & 71 & 67 & \\
\hline 7 & 4 & 1 & 3 & 4 & 2 & \\
\hline 8 & 4 & 1 & 31 & 25 & 3 & \\
\hline 9 & 4 & 1 & 31 & 26 & 2 & \\
\hline 10 & 4 & 22 & 26 & 31 & 25 & \\
\hline 11 & 4 & 2 & 26 & 22 & 4 & \\
\hline 12 & 4 & 3 & 25 & 22 & 4 & \\
\hline 13 & 4 & 5 & 32 & 27 & 6 & \\
\hline 14 & 4 & 5 & 32 & 28 & 7 & \\
\hline 15 & 4 & 5 & 7 & 8 & 6 & \\
\hline 16 & 4 & 7 & 28 & 23 & 8 & \\
\hline 17 & 4 & 6 & 27 & 23 & 8 & \\
\hline 18 & 4 & 23 & 28 & 32 & 27 & \\
\hline 19 & 4 & 9 & 33 & 29 & 10 & \\
\hline 20 & 4 & 9 & 11 & 12 & 10 & \\
\hline 21 & 4 & 9 & 33 & 30 & 11 & \\
\hline 22 & 4 & 11 & 30 & 24 & 12 & \\
\hline 23 & 4 & 24 & 30 & 33 & 29 & \\
\hline 24 & 4 & 10 & 29 & 24 & 12 & \\
\hline 25 & 5 & 53 & 63 & 67 & 70 & 59 \\
\hline 26 & 5 & 54 & 64 & 69 & 70 & 59 \\
\hline 27 & 4 & 50 & 54 & 59 & 53 & \\
\hline 28 & 4 & 62 & 66 & 67 & 63 & \\
\hline 29 & 4 & 62 & 66 & 69 & 64 & \\
\hline 30 & 3 & 50 & 62 & 63 & & \\
\hline 31 & 3 & 50 & 62 & 64 & & \\
\hline 32 & 5 & 2 & 13 & 14 & 15 & 4 \\
\hline 33 & 5 & 2 & 26 & 43 & 37 & 13 \\
\hline 34 & 4 & 13 & 37 & 34 & 14 & \\
\hline 35 & 4 & 4 & 22 & 40 & 15 & \\
\hline 36 & 4 & 22 & 40 & 43 & 26 & \\
\hline 37 & 3 & 14 & 34 & 40 & & \\
\hline 38 & 3 & 34 & 40 & 43 & & \\
\hline 39 & 5 & 6 & 27 & 44 & 38 & 16 \\
\hline 40 & 5 & 6 & 16 & 17 & 18 & 8 \\
\hline 41 & 4 & 16 & 38 & 35 & 17 & \\
\hline 42 & 4 & 23 & 41 & 44 & 27 & \\
\hline 43 & 4 & 8 & 23 & 41 & 18 & \\
\hline 44 & 3 & 35 & 41 & 44 & & \\
\hline 45 & 3 & 17 & 35 & 41 & & \\
\hline
\end{tabular}


Table 16 (Contd.)

\begin{tabular}{|c|c|c|c|c|c|c|}
\hline$i$ & $M$ & & & & & \\
\hline 46 & 5 & 29 & 45 & 55 & 60 & 33 \\
\hline 47 & 5 & 30 & 46 & 56 & 60 & 33 \\
\hline 48 & 4 & 51 & 56 & 60 & 55 & \\
\hline 49 & 4 & 24 & 42 & 45 & 29 & \\
\hline 50 & 4 & 24 & 42 & 46 & 30 & \\
\hline 51 & 3 & 42 & 51 & 55 & & \\
\hline 52 & 3 & 42 & 51 & 56 & & \\
\hline 53 & 5 & 57 & 65 & 68 & 71 & 61 \\
\hline 54 & 5 & 58 & 63 & 67 & 71 & 61 \\
\hline 55 & 4 & 52 & 58 & 61 & 57 & \\
\hline 56 & 4 & 62 & 66 & 68 & 65 & \\
\hline 57 & 3 & 52 & 62 & 65 & & \\
\hline 58 & 3 & 52 & 62 & 63 & & \\
\hline 59 & 5 & 3 & 25 & 47 & 39 & 19 \\
\hline 60 & 5 & 3 & 19 & 20 & 15 & 4 \\
\hline 61 & 4 & 19 & 39 & 36 & 20 & \\
\hline 62 & 4 & 22 & 40 & 47 & 25 & \\
\hline 63 & 3 & 36 & 40 & 47 & & \\
\hline 64 & 3 & 15 & 40 & 36 & & \\
\hline 65 & 5 & 28 & 48 & 58 & 61 & 32 \\
\hline 66 & 5 & 27 & 44 & 57 & 61 & 32 \\
\hline 67 & 4 & 23 & 41 & 48 & 28 & \\
\hline 68 & 3 & 41 & 52 & 58 & & \\
\hline 69 & 3 & 41 & 52 & 57 & & \\
\hline 70 & 5 & 10 & 19 & 20 & 21 & 12 \\
\hline 71 & 5 & 10 & 29 & 45 & 39 & 19 \\
\hline 72 & 4 & 12 & 24 & 42 & 21 & \\
\hline 73 & 3 & 20 & 36 & 42 & & \\
\hline 74 & 3 & 36 & 42 & 45 & & \\
\hline 75 & 5 & 55 & 64 & 69 & 72 & 60 \\
\hline 76 & 5 & 56 & 65 & 68 & 72 & 60 \\
\hline 77 & 3 & 51 & 62 & 64 & & \\
\hline 78 & 3 & 51 & 62 & 65 & & \\
\hline 79 & 5 & 26 & 43 & 53 & 59 & 31 \\
\hline 80 & 5 & 25 & 47 & 54 & 59 & 31 \\
\hline 81 & 3 & 40 & 50 & 53 & & \\
\hline 82 & 3 & 40 & 50 & 54 & & \\
\hline 83 & 5 & 7 & 13 & 14 & 18 & 8 \\
\hline 84 & 5 & 7 & 28 & 48 & 37 & 13 \\
\hline 85 & 3 & 14 & 34 & 41 & & \\
\hline 86 & 3 & 34 & 41 & 48 & & \\
\hline 87 & 5 & 11 & 30 & 46 & 38 & 16 \\
\hline 88 & 5 & 11 & 16 & 17 & 21 & 12 \\
\hline
\end{tabular}


Table 16 (Contd.)

\begin{tabular}{cc|cccccc}
\hline$i$ & $M$ & & & & & & \\
\hline 89 & 3 & 35 & 42 & 46 & & & \\
90 & 3 & 17 & 35 & 42 & & & \\
91 & 6 & 37 & 48 & 58 & 63 & 53 & 43 \\
92 & 3 & 49 & 62 & 50 & & & \\
93 & 3 & 49 & 62 & 52 & & & \\
94 & 3 & 40 & 50 & 49 & & & \\
95 & 3 & 34 & 49 & 40 & & & \\
96 & 3 & 34 & 49 & 41 & & & \\
97 & 3 & 41 & 52 & 49 & & & \\
98 & 6 & 14 & 18 & 17 & 21 & 20 & 15 \\
99 & 3 & 36 & 49 & 40 & & & \\
100 & 3 & 35 & 49 & 41 & & & \\
101 & 3 & 35 & 49 & 42 & & & \\
102 & 3 & 36 & 49 & 42 & & & \\
103 & 6 & 38 & 46 & 56 & 65 & 57 & 44 \\
104 & 3 & 42 & 51 & 49 & & & \\
105 & 3 & 49 & 62 & 51 & & & \\
106 & 6 & 39 & 47 & 54 & 64 & 55 & 45 \\
107 & 3 & 63 & 53 & 50 & & & \\
108 & 3 & 64 & 54 & 50 & & & \\
109 & 3 & 40 & 15 & 14 & & & \\
110 & 3 & 43 & 37 & 34 & & & \\
111 & 3 & 44 & 38 & 35 & & & \\
112 & 3 & 41 & 18 & 17 & & & \\
113 & 3 & 55 & 45 & 42 & & & \\
114 & 3 & 56 & 46 & 42 & & & \\
115 & 3 & 65 & 57 & 52 & & & \\
116 & 3 & 63 & 58 & 52 & & & \\
117 & 3 & 47 & 39 & 36 & & & \\
118 & 3 & 36 & 20 & 15 & & & \\
119 & 3 & 58 & 48 & 41 & & & \\
120 & 3 & 57 & 44 & 41 & & & \\
121 & 3 & 42 & 21 & 20 & & & \\
122 & 3 & 45 & 39 & 36 & & & \\
123 & 3 & 64 & 55 & 51 & & & \\
124 & 3 & 65 & 56 & 51 & & & \\
125 & 3 & 53 & 43 & 40 & & & \\
126 & 3 & 54 & 47 & 40 & & & \\
127 & 3 & 41 & 18 & 14 & & & \\
128 & 3 & 48 & 37 & 34 & & & \\
129 & 3 & 46 & 38 & 35 & & & \\
130 & 3 & 42 & 21 & 17 & & & \\
\hline & & & & & & \\
103 &
\end{tabular}


Table 17: The third order partition of the tetrahedron: the sub-cells.

\begin{tabular}{cc|cccccccccccc}
\hline$i$ & $M$ & \multicolumn{1}{c|}{} & & & & & & & & & & & \\
\hline 1 & 6 & 1 & 2 & 3 & 4 & 5 & 6 & & & & & \\
2 & 6 & 7 & 8 & 9 & 10 & 11 & 12 & & & & & \\
3 & 6 & 13 & 14 & 15 & 16 & 17 & 18 & & & & & \\
4 & 6 & 19 & 20 & 21 & 22 & 23 & 24 & & & & & \\
5 & 10 & 25 & 26 & 5 & 27 & 28 & 29 & 30 & 31 & 107 & 108 & & \\
6 & 10 & 32 & 33 & 11 & 34 & 35 & 36 & 37 & 38 & 109 & 110 & & \\
7 & 10 & 39 & 40 & 17 & 41 & 42 & 43 & 44 & 45 & 111 & 112 & & \\
8 & 10 & 46 & 47 & 23 & 48 & 49 & 50 & 51 & 52 & 113 & 114 & & \\
9 & 10 & 53 & 54 & 6 & 55 & 56 & 28 & 57 & 58 & 115 & 116 & & \\
10 & 10 & 59 & 60 & 12 & 61 & 62 & 35 & 63 & 64 & 117 & 118 & & \\
11 & 10 & 65 & 66 & 18 & 55 & 67 & 42 & 68 & 69 & 119 & 120 & & \\
12 & 10 & 70 & 71 & 24 & 61 & 72 & 49 & 73 & 74 & 121 & 122 & & \\
13 & 10 & 75 & 76 & 4 & 48 & 29 & 56 & 77 & 78 & 123 & 124 & & \\
14 & 10 & 79 & 80 & 10 & 27 & 36 & 62 & 81 & 82 & 125 & 126 & & \\
15 & 10 & 83 & 84 & 16 & 34 & 43 & 67 & 85 & 86 & 127 & 128 & & \\
16 & 10 & 87 & 88 & 22 & 41 & 50 & 72 & 89 & 90 & 129 & 130 & & \\
17 & 19 & 91 & 30 & 58 & 38 & 81 & 86 & 68 & 92 & 93 & 94 & 95 & 96 \\
& & 97 & 107 & 110 & 116 & 119 & 125 & 128 & & & & & \\
18 & 19 & 98 & 37 & 64 & 45 & 85 & 90 & 73 & 95 & 99 & 96 & 100 & 101 \\
& & 102 & 109 & 112 & 118 & 121 & 127 & 130 & & & & & \\
19 & 19 & 103 & 44 & 69 & 52 & 89 & 78 & 57 & 100 & 97 & 101 & 104 & 105 \\
& & 93 & 111 & 114 & 115 & 120 & 124 & 129 & & & & & \\
20 & 19 & 106 & 51 & 74 & 31 & 77 & 82 & 63 & 104 & 102 & 105 & 92 & 94 \\
& & 99 & 108 & 113 & 117 & 122 & 123 & 126 & & & & & \\
\hline
\end{tabular}

Table 18: The fourth order partition of the tetrahedron: the barycenter coordinates of the vertices.

\begin{tabular}{ccccc}
\hline$i$ & & & & \\
\hline 1 & 0.0000000000 & 1.0000000000 & 0.0000000000 & 0.0000000000 \\
2 & 0.0000000000 & 0.9400000000 & 0.0600000000 & 0.0000000000 \\
3 & 0.0000000000 & 0.9400000000 & 0.0000000000 & 0.0600000000 \\
4 & 0.0000000000 & 0.9200000000 & 0.0400000000 & 0.0400000000 \\
5 & 0.0000000000 & 0.0000000000 & 1.0000000000 & 0.0000000000 \\
6 & 0.0000000000 & 0.0000000000 & 0.9400000000 & 0.0600000000 \\
7 & 0.0000000000 & 0.0600000000 & 0.9400000000 & 0.0000000000 \\
8 & 0.0000000000 & 0.0400000000 & 0.9200000000 & 0.0400000000 \\
9 & 0.0000000000 & 0.0000000000 & 0.0000000000 & 1.0000000000 \\
10 & 0.0000000000 & 0.0600000000 & 0.0000000000 & 0.9400000000 \\
11 & 0.0000000000 & 0.0000000000 & 0.0600000000 & 0.9400000000 \\
12 & 0.0000000000 & 0.0400000000 & 0.0400000000 & 0.9200000000 \\
13 & 0.0000000000 & 0.6900000000 & 0.3100000000 & 0.0000000000 \\
\hline
\end{tabular}


Table 18 (Contd.)

\begin{tabular}{|c|c|c|c|c|}
\hline $\bar{i}$ & & & & \\
\hline 14 & 0.0000000000 & 0.7133333333 & 0.2466666667 & 0.0400000000 \\
\hline 15 & 0.0000000000 & 0.8400000000 & 0.0800000000 & 0.0800000000 \\
\hline 16 & 0.0000000000 & 0.0000000000 & 0.6900000000 & 0.3100000000 \\
\hline 17 & 0.0000000000 & 0.0400000000 & 0.7133333333 & 0.2466666667 \\
\hline 18 & 0.0000000000 & 0.0800000000 & 0.8400000000 & 0.0800000000 \\
\hline 19 & 0.0000000000 & 0.6900000000 & 0.0000000000 & 0.3100000000 \\
\hline 20 & 0.0000000000 & 0.7133333333 & 0.0400000000 & 0.2466666667 \\
\hline 21 & 0.0000000000 & 0.3100000000 & 0.0000000000 & 0.6900000000 \\
\hline 22 & 0.0000000000 & 0.24666666667 & 0.0400000000 & 0.7133333333 \\
\hline 23 & 0.0000000000 & 0.0800000000 & 0.0800000000 & 0.8400000000 \\
\hline 24 & 0.0000000000 & 0.3100000000 & 0.6900000000 & 0.0000000000 \\
\hline 25 & 0.0000000000 & 0.24666666667 & 0.7133333333 & 0.0400000000 \\
\hline 26 & 0.0000000000 & 0.0000000000 & 0.3100000000 & 0.6900000000 \\
\hline 27 & 0.0000000000 & 0.0400000000 & 0.2466666667 & 0.7133333333 \\
\hline 28 & 0.0000000000 & 0.4600000000 & 0.4600000000 & 0.0800000000 \\
\hline 29 & 0.0000000000 & 0.0800000000 & 0.4600000000 & 0.4600000000 \\
\hline 30 & 0.0000000000 & 0.4600000000 & 0.0800000000 & 0.4600000000 \\
\hline 31 & 0.0000000000 & 0.3333333333 & 0.3333333333 & 0.3333333333 \\
\hline 32 & 0.0300000000 & 0.9100000000 & 0.0300000000 & 0.0300000000 \\
\hline 33 & 0.0300000000 & 0.0300000000 & 0.9100000000 & 0.0300000000 \\
\hline 34 & 0.0300000000 & 0.0300000000 & 0.0300000000 & 0.9100000000 \\
\hline 35 & 0.0300000000 & 0.7250000000 & 0.2150000000 & 0.0300000000 \\
\hline 36 & 0.0300000000 & 0.0300000000 & 0.7250000000 & 0.2150000000 \\
\hline 37 & 0.0300000000 & 0.7250000000 & 0.0300000000 & 0.2150000000 \\
\hline 38 & 0.0300000000 & 0.2150000000 & 0.0300000000 & 0.7250000000 \\
\hline 39 & 0.0300000000 & 0.2150000000 & 0.7250000000 & 0.0300000000 \\
\hline 40 & 0.0300000000 & 0.0300000000 & 0.2150000000 & 0.7250000000 \\
\hline 41 & 0.0400000000 & 0.9200000000 & 0.0000000000 & 0.0400000000 \\
\hline 42 & 0.0400000000 & 0.9200000000 & 0.0400000000 & 0.0000000000 \\
\hline 43 & 0.0400000000 & 0.0000000000 & 0.9200000000 & 0.0400000000 \\
\hline 44 & 0.0400000000 & 0.0400000000 & 0.9200000000 & 0.0000000000 \\
\hline 45 & 0.0400000000 & 0.0400000000 & 0.0000000000 & 0.9200000000 \\
\hline 46 & 0.0400000000 & 0.0000000000 & 0.0400000000 & 0.9200000000 \\
\hline 47 & 0.0400000000 & 0.7133333333 & 0.2466666667 & 0.0000000000 \\
\hline 48 & 0.0400000000 & 0.0000000000 & 0.7133333333 & 0.2466666667 \\
\hline 49 & 0.0400000000 & 0.7133333333 & 0.0000000000 & 0.2466666667 \\
\hline 50 & 0.0400000000 & 0.24666666667 & 0.0000000000 & 0.7133333333 \\
\hline 51 & 0.0400000000 & 0.2466666667 & 0.7133333333 & 0.0000000000 \\
\hline 52 & 0.0400000000 & 0.0000000000 & 0.2466666667 & 0.7133333333 \\
\hline 53 & 0.0600000000 & 0.9400000000 & 0.0000000000 & 0.0000000000 \\
\hline 54 & 0.0600000000 & 0.0000000000 & 0.9400000000 & 0.0000000000 \\
\hline 55 & 0.0600000000 & 0.0000000000 & 0.0000000000 & 0.9400000000 \\
\hline 56 & 0.0600000000 & 0.8200000000 & 0.0600000000 & 0.0600000000 \\
\hline
\end{tabular}


Table 18 (Contd.)

\begin{tabular}{|c|c|c|c|c|}
\hline$\imath$ & & & & \\
\hline 57 & 0.0600000000 & 0.0600000000 & 0.8200000000 & 0.0600000000 \\
\hline 58 & 0.0600000000 & 0.0600000000 & 0.0600000000 & 0.8200000000 \\
\hline 59 & 0.0625000000 & 0.3125000000 & 0.3125000000 & 0.3125000000 \\
\hline 60 & 0.0800000000 & 0.8400000000 & 0.0800000000 & 0.0000000000 \\
\hline 61 & 0.0800000000 & 0.0000000000 & 0.8400000000 & 0.0800000000 \\
\hline 62 & 0.0800000000 & 0.0800000000 & 0.0000000000 & 0.8400000000 \\
\hline 63 & 0.0800000000 & 0.0000000000 & 0.0800000000 & 0.8400000000 \\
\hline 64 & 0.0800000000 & 0.8400000000 & 0.0000000000 & 0.0800000000 \\
\hline 65 & 0.0800000000 & 0.0800000000 & 0.8400000000 & 0.0000000000 \\
\hline 66 & 0.0800000000 & 0.4600000000 & 0.4600000000 & 0.0000000000 \\
\hline 67 & 0.0800000000 & 0.0000000000 & 0.4600000000 & 0.4600000000 \\
\hline 68 & 0.0800000000 & 0.4600000000 & 0.0000000000 & 0.4600000000 \\
\hline 69 & 0.0816666667 & 0.4183333333 & 0.4183333333 & 0.0816666667 \\
\hline 70 & 0.0816666667 & 0.0816666667 & 0.4183333333 & 0.4183333333 \\
\hline 71 & 0.0816666667 & 0.4183333333 & 0.0816666667 & 0.4183333333 \\
\hline 72 & 0.1225000000 & 0.6325000000 & 0.1225000000 & 0.1225000000 \\
\hline 73 & 0.1225000000 & 0.1225000000 & 0.6325000000 & 0.1225000000 \\
\hline 74 & 0.1225000000 & 0.1225000000 & 0.1225000000 & 0.6325000000 \\
\hline 75 & 0.2150000000 & 0.0300000000 & 0.0300000000 & 0.7250000000 \\
\hline 76 & 0.2150000000 & 0.0300000000 & 0.7250000000 & 0.0300000000 \\
\hline 77 & 0.2150000000 & 0.7250000000 & 0.0300000000 & 0.0300000000 \\
\hline 78 & 0.2466666667 & 0.0400000000 & 0.0000000000 & 0.7133333333 \\
\hline 79 & 0.2466666667 & 0.0000000000 & 0.0400000000 & 0.7133333333 \\
\hline 80 & 0.2466666667 & 0.0400000000 & 0.7133333333 & 0.0000000000 \\
\hline 81 & 0.2466666667 & 0.0000000000 & 0.7133333333 & 0.0400000000 \\
\hline 82 & 0.2466666667 & 0.7133333333 & 0.0400000000 & 0.0000000000 \\
\hline 83 & 0.2466666667 & 0.7133333333 & 0.0000000000 & 0.0400000000 \\
\hline 84 & 0.3100000000 & 0.0000000000 & 0.0000000000 & 0.6900000000 \\
\hline 85 & 0.3100000000 & 0.0000000000 & 0.6900000000 & 0.0000000000 \\
\hline 86 & 0.3100000000 & 0.6900000000 & 0.0000000000 & 0.0000000000 \\
\hline 87 & 0.3125000000 & 0.3125000000 & 0.3125000000 & 0.0625000000 \\
\hline 88 & 0.3125000000 & 0.0625000000 & 0.3125000000 & 0.3125000000 \\
\hline 89 & 0.3125000000 & 0.3125000000 & 0.0625000000 & 0.3125000000 \\
\hline 90 & 0.3333333333 & 0.3333333333 & 0.3333333333 & 0.0000000000 \\
\hline 91 & 0.3333333333 & 0.0000000000 & 0.3333333333 & 0.3333333333 \\
\hline 92 & 0.3333333333 & 0.3333333333 & 0.0000000000 & 0.3333333333 \\
\hline 93 & 0.4183333333 & 0.4183333333 & 0.0816666667 & 0.0816666667 \\
\hline 94 & 0.4183333333 & 0.0816666667 & 0.0816666667 & 0.4183333333 \\
\hline 95 & 0.4183333333 & 0.0816666667 & 0.4183333333 & 0.0816666667 \\
\hline 96 & 0.4600000000 & 0.4600000000 & 0.0800000000 & 0.0000000000 \\
\hline 97 & 0.4600000000 & 0.4600000000 & 0.0000000000 & 0.0800000000 \\
\hline 98 & 0.4600000000 & 0.0800000000 & 0.0000000000 & 0.4600000000 \\
\hline 99 & 0.4600000000 & 0.0000000000 & 0.0800000000 & 0.4600000000 \\
\hline
\end{tabular}


Table 18 (Contd.)

\begin{tabular}{ccccc}
\hline$i$ & & & & \\
\hline 100 & 0.4600000000 & 0.0000000000 & 0.4600000000 & 0.0800000000 \\
101 & 0.4600000000 & 0.0800000000 & 0.4600000000 & 0.0000000000 \\
102 & 0.6325000000 & 0.1225000000 & 0.1225000000 & 0.1225000000 \\
103 & 0.6900000000 & 0.3100000000 & 0.0000000000 & 0.0000000000 \\
104 & 0.6900000000 & 0.0000000000 & 0.3100000000 & 0.0000000000 \\
105 & 0.6900000000 & 0.0000000000 & 0.0000000000 & 0.3100000000 \\
106 & 0.7133333333 & 0.2466666667 & 0.0400000000 & 0.0000000000 \\
107 & 0.7133333333 & 0.2466666667 & 0.0000000000 & 0.0400000000 \\
108 & 0.7133333333 & 0.0000000000 & 0.2466666667 & 0.0400000000 \\
109 & 0.7133333333 & 0.0400000000 & 0.2466666667 & 0.0000000000 \\
110 & 0.7133333333 & 0.0400000000 & 0.0000000000 & 0.2466666667 \\
111 & 0.7133333333 & 0.0000000000 & 0.0400000000 & 0.2466666667 \\
112 & 0.7250000000 & 0.2150000000 & 0.0300000000 & 0.0300000000 \\
113 & 0.7250000000 & 0.0300000000 & 0.2150000000 & 0.0300000000 \\
114 & 0.7250000000 & 0.0300000000 & 0.0300000000 & 0.2150000000 \\
115 & 0.8200000000 & 0.0600000000 & 0.0600000000 & 0.0600000000 \\
116 & 0.8400000000 & 0.0800000000 & 0.0800000000 & 0.0000000000 \\
117 & 0.8400000000 & 0.0800000000 & 0.0000000000 & 0.0800000000 \\
118 & 0.8400000000 & 0.0000000000 & 0.0800000000 & 0.0800000000 \\
119 & 0.9100000000 & 0.0300000000 & 0.0300000000 & 0.0300000000 \\
120 & 0.9200000000 & 0.0400000000 & 0.0400000000 & 0.0000000000 \\
121 & 0.9200000000 & 0.0000000000 & 0.0400000000 & 0.0400000000 \\
122 & 0.9200000000 & 0.0400000000 & 0.0000000000 & 0.0400000000 \\
123 & 0.9400000000 & 0.0600000000 & 0.0000000000 & 0.0000000000 \\
124 & 0.9400000000 & 0.0000000000 & 0.0600000000 & 0.0000000000 \\
125 & 0.9400000000 & 0.0000000000 & 0.0000000000 & 0.0600000000 \\
126 & 1.0000000000 & 0.0000000000 & 0.0000000000 & 0.0000000000 \\
\hline & & & &
\end{tabular}

Table 19: The fourth order partition of the tetrahedron: the faces.

\begin{tabular}{cc|cccc}
\hline$i$ & $M$ & & & & \\
\hline 1 & 4 & 120 & 124 & 126 & 123 \\
2 & 4 & 121 & 125 & 126 & 124 \\
3 & 4 & 122 & 125 & 126 & 123 \\
4 & 4 & 119 & 122 & 125 & 121 \\
5 & 4 & 119 & 122 & 123 & 120 \\
6 & 4 & 119 & 121 & 124 & 120 \\
7 & 4 & 1 & 3 & 4 & 2 \\
8 & 4 & 1 & 53 & 41 & 3 \\
9 & 4 & 1 & 53 & 42 & 2 \\
10 & 4 & 32 & 42 & 53 & 41 \\
11 & 4 & 2 & 42 & 32 & 4 \\
\hline
\end{tabular}


Table 19 (Contd.)

\begin{tabular}{|c|c|c|c|c|c|c|}
\hline $\bar{i}$ & $\bar{M}$ & & & & & \\
\hline 12 & 4 & 3 & 41 & 32 & 4 & \\
\hline 13 & 4 & 5 & 54 & 43 & 6 & \\
\hline 14 & 4 & 5 & 54 & 44 & 7 & \\
\hline 15 & 4 & 5 & 7 & 8 & 6 & \\
\hline 16 & 4 & 7 & 44 & 33 & 8 & \\
\hline 17 & 4 & 6 & 43 & 33 & 8 & \\
\hline 18 & 4 & 33 & 44 & 54 & 43 & \\
\hline 19 & 4 & 9 & 55 & 45 & 10 & \\
\hline 20 & 4 & 9 & 11 & 12 & 10 & \\
\hline 21 & 4 & 9 & 55 & 46 & 11 & \\
\hline 22 & 4 & 11 & 46 & 34 & 12 & \\
\hline 23 & 4 & 34 & 46 & 55 & 45 & \\
\hline 24 & 4 & 10 & 45 & 34 & 12 & \\
\hline 25 & 5 & 103 & 123 & 120 & 116 & 106 \\
\hline 26 & 5 & 103 & 123 & 122 & 117 & 107 \\
\hline 27 & 4 & 103 & 107 & 112 & 106 & \\
\hline 28 & 4 & 115 & 119 & 120 & 116 & \\
\hline 29 & 4 & 115 & 119 & 122 & 117 & \\
\hline 30 & 3 & 106 & 116 & 115 & & \\
\hline 31 & 3 & 107 & 117 & 115 & & \\
\hline 32 & 5 & 2 & 13 & 14 & 15 & 4 \\
\hline 33 & 5 & 2 & 42 & 60 & 47 & 13 \\
\hline 34 & 4 & 13 & 47 & 35 & 14 & \\
\hline 35 & 4 & 4 & 32 & 56 & 15 & \\
\hline 36 & 4 & 32 & 56 & 60 & 42 & \\
\hline 37 & 3 & 14 & 35 & 56 & & \\
\hline 38 & 3 & 35 & 56 & 60 & & \\
\hline 39 & 5 & 6 & 43 & 61 & 48 & 16 \\
\hline 40 & 5 & 6 & 16 & 17 & 18 & 8 \\
\hline 41 & 4 & 16 & 48 & 36 & 17 & \\
\hline 42 & 4 & 33 & 57 & 61 & 43 & \\
\hline 43 & 4 & 8 & 33 & 57 & 18 & \\
\hline 44 & 3 & 36 & 57 & 61 & & \\
\hline 45 & 3 & 17 & 36 & 57 & & \\
\hline 46 & 5 & 45 & 62 & 78 & 84 & 55 \\
\hline 47 & 5 & 46 & 63 & 79 & 84 & 55 \\
\hline 48 & 4 & 75 & 79 & 84 & 78 & \\
\hline 49 & 4 & 34 & 58 & 62 & 45 & \\
\hline 50 & 4 & 34 & 58 & 63 & 46 & \\
\hline 51 & 3 & 58 & 75 & 78 & & \\
\hline 52 & 3 & 58 & 75 & 79 & & \\
\hline 53 & 5 & 104 & 124 & 121 & 118 & 108 \\
\hline 54 & 5 & 104 & 124 & 120 & 116 & 109 \\
\hline
\end{tabular}


Table 19 (Contd.)

\begin{tabular}{|c|c|c|c|c|c|c|}
\hline $\bar{i}$ & $\bar{M}$ & & & & & \\
\hline 55 & 4 & 104 & 109 & 113 & 108 & \\
\hline 56 & 4 & 115 & 119 & 121 & 118 & \\
\hline 57 & 3 & 108 & 118 & 115 & & \\
\hline 58 & 3 & 109 & 116 & 115 & & \\
\hline 59 & 5 & 3 & 41 & 64 & 49 & 19 \\
\hline 60 & 5 & 3 & 19 & 20 & 15 & 4 \\
\hline 61 & 4 & 19 & 49 & 37 & 20 & \\
\hline 62 & 4 & 32 & 56 & 64 & 41 & \\
\hline 63 & 3 & 37 & 56 & 64 & & \\
\hline 64 & 3 & 15 & 56 & 37 & & \\
\hline 65 & 5 & 44 & 65 & 80 & 85 & 54 \\
\hline 66 & 5 & 43 & 61 & 81 & 85 & 54 \\
\hline 67 & 4 & 76 & 81 & 85 & 80 & \\
\hline 68 & 4 & 33 & 57 & 65 & 44 & \\
\hline 69 & 3 & 57 & 76 & 80 & & \\
\hline 70 & 3 & 57 & 76 & 81 & & \\
\hline 71 & 5 & 10 & 21 & 22 & 23 & 12 \\
\hline 72 & 5 & 10 & 45 & 62 & 50 & 21 \\
\hline 73 & 4 & 21 & 50 & 38 & 22 & \\
\hline 74 & 4 & 12 & 34 & 58 & 23 & \\
\hline 75 & 3 & 22 & 38 & 58 & & \\
\hline 76 & 3 & 38 & 58 & 62 & & \\
\hline 77 & 5 & 105 & 125 & 122 & 117 & 110 \\
\hline 78 & 5 & 105 & 125 & 121 & 118 & 111 \\
\hline 79 & 4 & 105 & 111 & 114 & 110 & \\
\hline 80 & 3 & 110 & 117 & 115 & & \\
\hline 81 & 3 & 111 & 118 & 115 & & \\
\hline 82 & 5 & 42 & 60 & 82 & 86 & 53 \\
\hline 83 & 5 & 41 & 64 & 83 & 86 & 53 \\
\hline 84 & 4 & 77 & 83 & 86 & 82 & \\
\hline 85 & 3 & 56 & 77 & 82 & & \\
\hline 86 & 3 & 56 & 77 & 83 & & \\
\hline 87 & 5 & 7 & 24 & 25 & 18 & 8 \\
\hline 88 & 5 & 7 & 44 & 65 & 51 & 24 \\
\hline 89 & 4 & 24 & 51 & 39 & 25 & \\
\hline 90 & 3 & 18 & 57 & 39 & & \\
\hline 91 & 3 & 39 & 57 & 65 & & \\
\hline 92 & 5 & 11 & 46 & 63 & 52 & 26 \\
\hline 93 & 5 & 11 & 26 & 27 & 23 & 12 \\
\hline 94 & 4 & 26 & 52 & 40 & 27 & \\
\hline 95 & 3 & 40 & 58 & 63 & & \\
\hline 96 & 3 & 23 & 58 & 40 & & \\
\hline 97 & 5 & 82 & 96 & 106 & 103 & 86 \\
\hline
\end{tabular}


Table 19 (Contd.)

\begin{tabular}{|c|c|c|c|c|c|c|c|}
\hline $\bar{i}$ & $\bar{M}$ & & & & & & \\
\hline 98 & 5 & 83 & 97 & 107 & 103 & 86 & \\
\hline 99 & 3 & 77 & 93 & 96 & & & \\
\hline 100 & 3 & 77 & 93 & 97 & & & \\
\hline 101 & 3 & 93 & 112 & 106 & & & \\
\hline 102 & 3 & 93 & 112 & 107 & & & \\
\hline 103 & 5 & 13 & 24 & 25 & 28 & 14 & \\
\hline 104 & 5 & 13 & 47 & 66 & 51 & 24 & \\
\hline 105 & 3 & 25 & 39 & 69 & & & \\
\hline 106 & 3 & 39 & 69 & 66 & & & \\
\hline 107 & 3 & 14 & 35 & 69 & & & \\
\hline 108 & 3 & 35 & 69 & 66 & & & \\
\hline 109 & 5 & 16 & 48 & 67 & 52 & 26 & \\
\hline 110 & 5 & 16 & 26 & 27 & 29 & 17 & \\
\hline 111 & 3 & 40 & 70 & 67 & & & \\
\hline 112 & 3 & 27 & 40 & 70 & & & \\
\hline 113 & 3 & 36 & 70 & 67 & & & \\
\hline 114 & 3 & 17 & 36 & 70 & & & \\
\hline 115 & 5 & 78 & 98 & 110 & 105 & 84 & \\
\hline 116 & 5 & 79 & 99 & 111 & 105 & 84 & \\
\hline 117 & 3 & 94 & 114 & 110 & & & \\
\hline 118 & 3 & 94 & 114 & 111 & & & \\
\hline 119 & 3 & 75 & 94 & 98 & & & \\
\hline 120 & 3 & 75 & 94 & 99 & & & \\
\hline 121 & 5 & 81 & 100 & 108 & 104 & 85 & \\
\hline 122 & 5 & 80 & 101 & 109 & 104 & 85 & \\
\hline 123 & 3 & 76 & 95 & 100 & & & \\
\hline 124 & 3 & 76 & 95 & 101 & & & \\
\hline 125 & 3 & 95 & 113 & 108 & & & \\
\hline 126 & 3 & 95 & 113 & 109 & & & \\
\hline 127 & 5 & 19 & 49 & 68 & 50 & 21 & \\
\hline 128 & 5 & 19 & 21 & 22 & 30 & 20 & \\
\hline 129 & 3 & 38 & 71 & 68 & & & \\
\hline 130 & 3 & 22 & 38 & 71 & & & \\
\hline 131 & 3 & 37 & 71 & 68 & & & \\
\hline 132 & 3 & 20 & 37 & 71 & & & \\
\hline 133 & 6 & 90 & 101 & 109 & 116 & 106 & 96 \\
\hline 134 & 4 & 87 & 93 & 96 & 90 & & \\
\hline 135 & 4 & 87 & 95 & 101 & 90 & & \\
\hline 136 & 3 & 102 & 115 & 112 & & & \\
\hline 137 & 3 & 102 & 115 & 113 & & & \\
\hline 138 & 3 & 93 & 112 & 102 & & & \\
\hline 139 & 3 & 87 & 102 & 93 & & & \\
\hline 140 & 3 & 87 & 102 & 95 & & & \\
\hline
\end{tabular}


Table 19 (Contd.)

\begin{tabular}{cc|cccccc}
\hline$i$ & $M$ & & & & & & \\
\hline 141 & 3 & 95 & 113 & 102 & & & \\
142 & 6 & 14 & 28 & 31 & 30 & 20 & 15 \\
143 & 4 & 28 & 69 & 59 & 31 & & \\
144 & 4 & 30 & 71 & 59 & 31 & & \\
145 & 3 & 35 & 72 & 56 & & & \\
146 & 3 & 37 & 72 & 56 & & & \\
147 & 3 & 35 & 72 & 69 & & & \\
148 & 3 & 59 & 72 & 69 & & & \\
149 & 3 & 59 & 72 & 71 & & & \\
150 & 3 & 37 & 72 & 71 & & & \\
151 & 6 & 48 & 67 & 91 & 100 & 81 & 61 \\
152 & 4 & 67 & 91 & 88 & 70 & & \\
153 & 4 & 88 & 95 & 100 & 91 & & \\
154 & 3 & 36 & 73 & 57 & & & \\
155 & 3 & 57 & 76 & 73 & & & \\
156 & 3 & 36 & 73 & 70 & & & \\
157 & 3 & 70 & 88 & 73 & & & \\
158 & 3 & 73 & 95 & 88 & & & \\
159 & 3 & 73 & 95 & 76 & & & \\
160 & 6 & 50 & 68 & 92 & 98 & 78 & 62 \\
161 & 4 & 89 & 94 & 98 & 92 & & \\
162 & 4 & 68 & 92 & 89 & 71 & & \\
163 & 3 & 58 & 75 & 74 & & & \\
164 & 3 & 38 & 74 & 58 & & & \\
165 & 3 & 74 & 94 & 75 & & & \\
166 & 3 & 74 & 94 & 89 & & & \\
167 & 3 & 71 & 89 & 74 & & & \\
168 & 3 & 38 & 74 & 71 & & & \\
169 & 6 & 91 & 100 & 108 & 118 & 111 & 99 \\
170 & 4 & 88 & 94 & 99 & 91 & & \\
171 & 3 & 102 & 115 & 114 & & & \\
172 & 3 & 88 & 102 & 95 & & & \\
173 & 3 & 88 & 102 & 94 & & & \\
174 & 3 & 94 & 114 & 102 & & & \\
175 & 6 & 49 & 68 & 92 & 97 & 83 & 64 \\
176 & 4 & 89 & 93 & 97 & 92 & & \\
177 & 3 & 56 & 77 & 72 & & & \\
178 & 3 & 71 & 89 & 72 & & & \\
179 & 3 & 72 & 93 & 89 & & & \\
180 & 3 & 72 & 93 & 77 & & & \\
181 & 6 & 51 & 66 & 90 & 101 & 80 & 65 \\
182 & 4 & 66 & 90 & 87 & 69 & & \\
183 & 3 & 39 & 73 & 57 & & & \\
\hline & & & & & & & \\
153 &
\end{tabular}


Table 19 (Contd.)

\begin{tabular}{cc|cccccc}
\hline$i$ & $M$ & & & & & & \\
\hline 184 & 3 & 73 & 95 & 87 & & & \\
185 & 3 & 69 & 87 & 73 & & & \\
186 & 3 & 39 & 73 & 69 & & & \\
187 & 6 & 22 & 30 & 31 & 29 & 27 & 23 \\
188 & 4 & 29 & 70 & 59 & 31 & & \\
189 & 3 & 40 & 74 & 58 & & & \\
190 & 3 & 59 & 74 & 71 & & & \\
191 & 3 & 59 & 74 & 70 & & & \\
192 & 3 & 40 & 74 & 70 & & & \\
193 & 6 & 92 & 98 & 110 & 117 & 107 & 97 \\
194 & 3 & 89 & 102 & 94 & & & \\
195 & 3 & 89 & 102 & 93 & & & \\
196 & 6 & 47 & 66 & 90 & 96 & 82 & 60 \\
197 & 3 & 72 & 93 & 87 & & & \\
198 & 3 & 69 & 87 & 72 & & & \\
199 & 6 & 17 & 29 & 31 & 28 & 25 & 18 \\
200 & 3 & 59 & 73 & 69 & & & \\
201 & 3 & 59 & 73 & 70 & & & \\
202 & 6 & 52 & 67 & 91 & 99 & 79 & 63 \\
203 & 3 & 70 & 88 & 74 & & & \\
204 & 3 & 74 & 94 & 88 & & & \\
205 & 3 & 115 & 112 & 106 & & & \\
206 & 3 & 115 & 112 & 107 & & & \\
207 & 3 & 56 & 15 & 14 & & & \\
208 & 3 & 60 & 47 & 35 & & & \\
209 & 3 & 61 & 48 & 36 & & & \\
210 & 3 & 57 & 18 & 17 & & & \\
211 & 3 & 78 & 62 & 58 & & & \\
212 & 3 & 79 & 63 & 58 & & & \\
213 & 3 & 115 & 113 & 108 & & & \\
214 & 3 & 115 & 113 & 109 & & & \\
215 & 3 & 64 & 49 & 37 & & & \\
216 & 3 & 37 & 20 & 15 & & & \\
217 & 3 & 80 & 65 & 57 & & & \\
218 & 3 & 81 & 61 & 57 & & & \\
219 & 3 & 58 & 23 & 22 & & & \\
220 & 3 & 62 & 50 & 38 & & & \\
221 & 3 & 115 & 114 & 110 & & & \\
222 & 3 & 115 & 114 & 111 & & & \\
223 & 3 & 82 & 60 & 56 & & & \\
224 & 3 & 83 & 64 & 56 & & & \\
225 & 3 & 39 & 25 & 18 & & & \\
226 & 3 & 65 & 51 & 39 & & & \\
\hline & & & & & & & \\
193 &
\end{tabular}


Table 19 (Contd.)

\begin{tabular}{cc|ccc}
\hline$i$ & $M$ & & & \\
\hline 227 & 3 & 63 & 52 & 40 \\
228 & 3 & 40 & 27 & 23 \\
229 & 3 & 96 & 82 & 77 \\
230 & 3 & 97 & 83 & 77 \\
231 & 3 & 106 & 96 & 93 \\
232 & 3 & 107 & 97 & 93 \\
233 & 3 & 69 & 28 & 25 \\
234 & 3 & 66 & 51 & 39 \\
235 & 3 & 69 & 28 & 14 \\
236 & 3 & 66 & 47 & 35 \\
237 & 3 & 67 & 52 & 40 \\
238 & 3 & 70 & 29 & 27 \\
239 & 3 & 67 & 48 & 36 \\
240 & 3 & 70 & 29 & 17 \\
241 & 3 & 110 & 98 & 94 \\
242 & 3 & 111 & 99 & 94 \\
243 & 3 & 98 & 78 & 75 \\
244 & 3 & 99 & 79 & 75 \\
245 & 3 & 100 & 81 & 76 \\
246 & 3 & 101 & 80 & 76 \\
247 & 3 & 108 & 100 & 95 \\
248 & 3 & 109 & 101 & 95 \\
249 & 3 & 68 & 50 & 38 \\
250 & 3 & 71 & 30 & 22 \\
251 & 3 & 68 & 49 & 37 \\
252 & 3 & 71 & 30 & 20 \\
\hline
\end{tabular}

Table 20: The fourth order partition of the tetrahedron: the sub-cells.

\begin{tabular}{|c|c|c|c|c|c|c|c|c|c|c|c|}
\hline $\bar{i}$ & $M$ & & & & & & & & & & \\
\hline 1 & 6 & 1 & 2 & 3 & 4 & 5 & 6 & & & & \\
\hline 2 & 6 & 7 & 8 & 9 & 10 & 11 & 12 & & & & \\
\hline 3 & 6 & 13 & 14 & 15 & 16 & 17 & 18 & & & & \\
\hline 4 & 6 & 19 & 20 & 21 & 22 & 23 & 24 & & & & \\
\hline 5 & 10 & 25 & 26 & 5 & 27 & 28 & 29 & 30 & 31 & 205 & 206 \\
\hline 6 & 10 & 32 & 33 & 11 & 34 & 35 & 36 & 37 & 38 & 207 & 208 \\
\hline 7 & 10 & 39 & 40 & 17 & 41 & 42 & 43 & 44 & 45 & 209 & 210 \\
\hline 8 & 10 & 46 & 47 & 23 & 48 & 49 & 50 & 51 & 52 & 211 & 212 \\
\hline 9 & 10 & 53 & 54 & 6 & 55 & 56 & 28 & 57 & 58 & 213 & 214 \\
\hline 10 & 10 & 59 & 60 & 12 & 61 & 62 & 35 & 63 & 64 & 215 & 216 \\
\hline 11 & 10 & 65 & 66 & 18 & 67 & 68 & 42 & 69 & 70 & 217 & 218 \\
\hline 12 & 10 & 71 & 72 & 24 & 73 & 74 & 49 & 75 & 76 & 219 & 220 \\
\hline
\end{tabular}


Table 20 (Contd.)

\begin{tabular}{|c|c|c|c|c|c|c|c|c|c|c|c|c|c|}
\hline$i$ & $M$ & & & & & & & & & & & & \\
\hline 13 & 10 & 77 & 78 & 4 & 79 & 29 & 56 & 80 & 81 & 221 & 222 & & \\
\hline 14 & 10 & 82 & 83 & 10 & 84 & 36 & 62 & 85 & 86 & 223 & 224 & & \\
\hline 15 & 10 & 87 & 88 & 16 & 89 & 43 & 68 & 90 & 91 & 225 & 226 & & \\
\hline 16 & 10 & 92 & 93 & 22 & 94 & 50 & 74 & 95 & 96 & 227 & 228 & & \\
\hline 17 & 12 & 97 & 98 & 84 & 27 & 99 & 100 & 101 & 102 & 229 & 230 & 231 & 232 \\
\hline 18 & 12 & 103 & 04 & 89 & 34 & 105 & 06 & 107 & 08 & 233 & 234 & 5 & \\
\hline 19 & 12 & 109 & 110 & 94 & 41 & 111 & 112 & 113 & 114 & 237 & 238 & 9 & 0 \\
\hline 20 & 12 & 115 & 116 & 79 & 48 & 117 & 118 & 119 & 120 & 241 & 242 & 243 & 244 \\
\hline 21 & 12 & 1 & 122 & 67 & 55 & 123 & 124 & 125 & 126 & 245 & 246 & 247 & 248 \\
\hline 22 & 12 & 27 & 128 & 73 & 61 & 29 & 130 & 131 & 132 & 249 & 250 & 251 & 252 \\
\hline \multirow[t]{2}{*}{23} & 17 & 133 & 30 & 58 & 34 & 101 & 135 & 126 & 136 & 137 & 138 & & 12 \\
\hline & & 141 & 205 & 214 & 231 & 248 & & & & & & & \\
\hline \multirow[t]{2}{*}{24} & 17 & 142 & 37 & 64 & 143 & 107 & 144 & 132 & 145 & 146 & 147 & 148 & 149 \\
\hline & & 15 & 207 & 16 & 235 & 252 & & & & & & & \\
\hline \multirow[t]{2}{*}{25} & 17 & 15 & 44 & 70 & 152 & 113 & 153 & 123 & 154 & 155 & 156 & 15 & 15 \\
\hline & & 159 & 209 & 218 & 239 & 245 & & & & & & & \\
\hline \multirow[t]{2}{*}{26} & 17 & 160 & 51 & 76 & 161 & 119 & 162 & 129 & 163 & 164 & 165 & 166 & 167 \\
\hline & & 16 & 211 & 220 & 243 & 249 & & & & & & & \\
\hline \multirow[t]{2}{*}{27} & 17 & 169 & 57 & 81 & 153 & 125 & 170 & 118 & 137 & 171 & 141 & 17 & 173 \\
\hline & & 174 & 213 & 222 & 242 & 247 & & & & & & & \\
\hline \multirow[t]{2}{*}{28} & 17 & 175 & 63 & 86 & 162 & 131 & 176 & 100 & 146 & 177 & 150 & 178 & 179 \\
\hline & & 18 & 21 & 224 & 23 & 251 & & & & & & & \\
\hline \multirow[t]{2}{*}{29} & 17 & 18 & 69 & 91 & 1. & 124 & 182 & 106 & 155 & 183 & 159 & 184 & 185 \\
\hline & & 18 & 217 & 226 & 234 & 246 & & & & & & & \\
\hline \multirow[t]{2}{*}{30} & 17 & 18 & 75 & 96 & 144 & 130 & 188 & 112 & 164 & 189 & 168 & 190 & 191 \\
\hline & & 19 & 219 & 228 & 238 & 250 & & & & & & & \\
\hline \multirow[t]{2}{*}{31} & 17 & 19 & 80 & 31 & & 117 & 176 & 102 & 171 & 136 & 174 & 194 & 195 \\
\hline & & 13 & 206 & 221 & 23 & 24 & & & & & & & \\
\hline \multirow[t]{2}{*}{32} & 17 & 196 & 85 & 38 & 134 & 99 & 182 & 108 & 177 & 145 & 180 & 197 & 198 \\
\hline & & 14 & 208 & 223 & 229 & 236 & & & & & & & \\
\hline \multirow[t]{2}{*}{33} & 17 & 19 & 90 & 45 & 12 & 105 & 188 & 11 & 183 & 154 & 186 & 200 & 201 \\
\hline & & 15 & 210 & 22 & $2{ }^{2}$ & 22 & & & & & & & \\
\hline \multirow[t]{2}{*}{34} & 17 & 202 & 95 & 52 & 15 & 111 & 170 & 120 & 189 & 163 & 192 & 203 & 204 \\
\hline & & 165 & 212 & 227 & 237 & 244 & & & & & & & \\
\hline \multirow[t]{2}{*}{35} & 24 & 194 & 173 & 19 & 139 & 140 & 17 & 197 & 17 & 19 & 14 & & \\
\hline & & 200 & 185 & 201 & 157 & 158 & 184 & 203 & 191 & 204 & 166 & 167 & 190 \\
\hline
\end{tabular}




\section{References}

[1] Q. Chen and I. Babuška. Approximate optimal points for polynomial interpolation of real functions in an interval and in a triangle. Computer Methods in Applied Mechanics and Engineering, 128:405-417, 1995.

[2] Q. Chen and I. Babuška. The optimal symmetrical points for polynomial interpolation of real functions in the tetrahedron. Computer Methods in Applied Mechanics and Engineering, 137:89-94, 1996.

[3] Q.-Y. Chen. Partitions of a simplex leading to accurate spectral (finite) volume reconstruction. SIAM Journal on Scientific Computing. To Appear.

[4] P. J. Davis. Interpolation and Approximation. Dover Publications, New York, 1975.

[5] J. S. Hesthaven. From electrostatics to almost optimal nodal sets for polynomial interpolation in a simplex. SIAM Journal on Numerical Analysis, 35(2):655-676, 1998.

[6] J. S. Hesthaven and C. H. Teng. Stable spectral methods on tetrahedral elements. SIAM Journal on Scientific Computing, 21(6):2352-2380, 2000.

[7] Y. Liu, M. Vinokur, and Z. Wang. Three-dimensional high-order spectral finite volume method for unstructured grids. In Proceedings of 16th AIAA CFD Conference, Orlando, Florida, 2003. AIAA Paper No. 2003-3837.

[8] M. A. Taylor, B. A. Wingate, and R. E. Vincent. An algorithm for computing fekete points in the triangle. SIAM Journal On Numerical Analysis, 38(5):1707-1720, 2000.

[9] Z. Wang and Y. Liu. Spectral (finite) volume method for conservation laws on unstructured grids iii: One dimensional systems and partition optimization. Journal of Scientific Computing, 20(1):137-157, 2004.

[10] Z. J. Wang. Spectral (finite) volume method for conservation laws on unstructured grids: Basic formulation. Journal of Computational Physics, 178:210-251, 2002. 
[11] Z. J. Wang and Y. Liu. Spectral(finite) volume method for conservation laws on unstructured grids. Journal of Computational Physics, 179:665-697, 2002. 